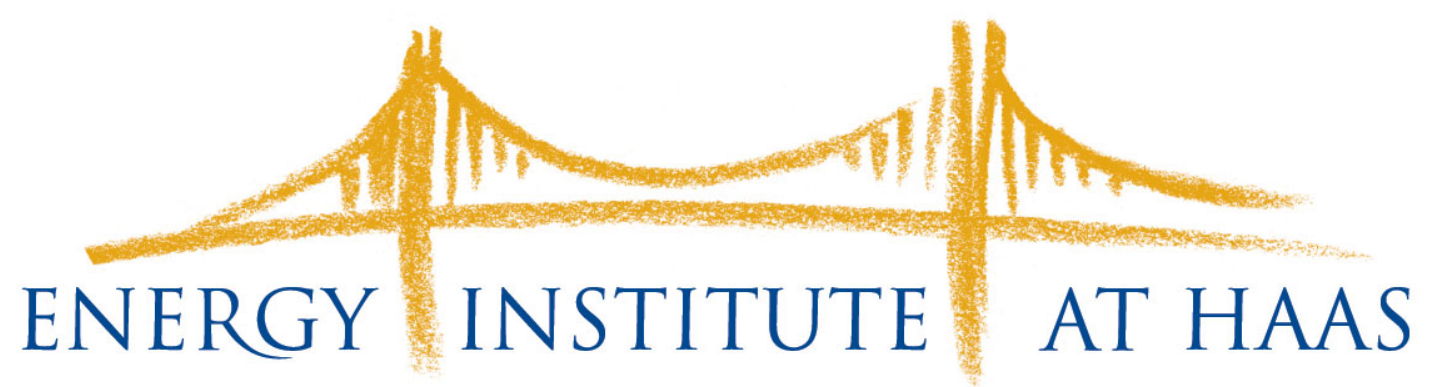

EI @ Haas WP 277

\title{
Panel Data and Experimental Design
}

\section{Fiona Burlig, Louis Preonas, and Matt Woerman}

\author{
January 2017
}

Energy Institute at Haas working papers are circulated for discussion and comment purposes. They have not been peer-reviewed or been subject to review by any editorial board.

(C) 2017 by Fiona Burlig, Louis Preonas, and Matt Woerman. All rights reserved. Short sections of text, not to exceed two paragraphs, may be quoted without explicit permission provided that full credit is given to the source.

http://ei.haas.berkeley.edu 


\title{
Panel Data and Experimental Design
}

\author{
Fiona Burlig, Louis Preonas, and Matt Woerman*
}

January 2, 2017

\begin{abstract}
How should researchers design experiments to detect treatment effects with panel data? In this paper, we derive analytical expressions for the variance of panel estimators under non-i.i.d. error structures, which inform power calculations in panel data settings. Using Monte Carlo simulation, we demonstrate that, with correlated errors, traditional methods for experimental design result in experiments that are incorrectly powered with proper inference. Failing to account for serial correlation yields overpowered experiments in short panels and underpowered experiments in long panels. Using both data from a randomized experiment in China and a high-frequency dataset of U.S. electricity consumption, we show that these results hold in real-world settings. Our theoretical results enable us to achieve correctly powered experiments in both simulated and real data. This paper provides researchers with the tools to design well-powered experiments in panel data settings.
\end{abstract}

Keywords: power, experimental design, panel data

${ }^{*}$ University of California, Berkeley: Department of Agricultural and Resource Economics and Energy Institute at Haas. 207 Giannini Hall, UC Berkeley, Berkeley, CA 94720. Burlig: fiona.burlig@berkeley.edu. Preonas: lpreonas@berkeley.edu. Woerman: matt. woerman@berkeley.edu We thank Michael Anderson, Maximilian Auffhammer, Patrick Baylis, Joshua Blonz, Severin Borenstein, HaiAnh Dang, Meredith Fowlie, James Gillan, Jeremy Magruder, Aprajit Mahajan, Edward Miguel, Tavneet Suri, Catherine Wolfram, and seminar participants at the University of California, Berkeley and NEUDC for valuable comments. Molly Van Dop provided excellent research assistance. Funding for this research was provided by the Berkeley Initiative for Transparency in the Social Sciences, a program of the Center for Effective Global Action (CEGA), with support from the Laura and John Arnold Foundation. Burlig is generously supported by the National Science Foundation's Graduate Research Fellowship Program under grant DGE-1106400. All remaining errors are our own. Our online appendix is available at https://ei.haas.berkeley.edu/research/papers/WP277Appendix.pdf Our accompanying STATA package, pcpanel, is available from ssc; an $\mathrm{R}$ version will follow shortly, which will be available from CRAN. 


\section{Introduction}

Randomized controlled trials (RCTs) are an extremely valuable and increasingly popular tool for causal inference. The number of RCTs published in the top five economics journals has risen substantially over time (Card, DellaVigna, and Malmendier (2011)). As researchers embark on RCTs, they face many challenges in designing experiments: they must choose a sampling frame and sample size, design an intervention, and collect data, all subject to budget constraints. Experiments must have large enough sample sizes to be sufficiently powered, or to be able to statistically distinguish between true and false null hypotheses. At the same time, their sample sizes must be small enough to keep costs down.

Power calculations represent an important tool for calibrating the sample size and design of RCTs. By applying either analytical formulas or simulation-based algorithms, power calculations enable researchers to trade off sample size with the smallest effect an experiment can empirically detect. Bloom (1995) provides an early overview of the power calculation framework.1. Duflo, Glennerster, and Kremer 2007) and Glennerster and Takavarashi (2013) describe the basics of power calculations and discuss practical considerations. The existing literature on statistical power in economics focuses on single-wave experiments, where units are randomized into a treatment group or a control group, and researchers observe each unit once $2^{2}$

In a widely cited paper based on results from Frison and Pocock (1992), McKenzie (2012) recommends considering experimental designs that involve panel data, using multiple observations per unit to increase statistical power. This is especially attractive in settings where collecting additional waves of data for one individual is more cost-effective than collecting data on more individuals. In recent years, several prominent papers have employed RCT designs with panel data. $3^{3}$ As data collection becomes cheaper and easier, panel RCTs are becoming increasingly common, allowing researchers to answer new questions using more flexible empirical strategies.

Panel data also poses challenges in terms of statistical inference. Bertrand, Duflo, and Mullainathan (2004) highlights the notion that units in panel data generally exhibit serial correlation,

1. Cohen (1977) and Murphy, Myors, and Wolach (2014) are classic references.

2. In economics, researchers often collect two waves of data, but estimate treatment effects using post-treatment data only and controlling for the baseline level of the outcome variable (following McKenzie (2012)). Baird et al. (2014) extends the classic cross-sectional setup to randomized saturation designs, capable of measuring spillover and general equilibrium effects. Athey and Imbens (2016a) discusses statistical power using a randomization inference approach.

3. These include Bloom et al. (2013), Blattman, Fiala, and Martinez (2014), Jessoe and Rapson (2014), Bloom et al. (2015), Fowlie, Greenstone, and Wolfram (2015), and Fowlie et al. (2016). 
and that failing to account for this error structure will yield standard errors that are biased towards zero. This dramatically raises the probability of a Type I error. In order to achieve correct false rejection rates, applied econometricians using panel data in quasi-experimental settings generally implement the cluster-robust variance estimator (CRVE), or use "clustered standard errors": 4

In a panel RCT, it is likewise important to account for serially correlated errors both during $e x$ post analysis and in ex ante experimental design. If researchers assume that errors are independent and identically distributed (i.i.d.) in ex ante power calculations, and then do not adjust their standard errors ex post, they will over-reject true null hypotheses if their errors are in fact serially correlated. On the other hand, if researchers adjust their standard errors ex post but do not adjust their power calculations ex ante, they introduce a fundamental mismatch between ex ante and ex post assumptions that will yield incorrectly powered experiments in the presence of serial correlation. To the best of our knowledge, there is no existing economics literature on power calculations in panel data that accounts for arbitrary serial correlation.

In this paper, we derive analytical expressions for the variance of panel estimators under non-i.i.d. error structures. We use these expressions to formalize a power calculation formula for difference-in-differences estimators that is robust to serial correlation in panel data settings. We conduct Monte Carlo analysis using both simulated and real data, and demonstrate that standard methods for experimental design yield experiments that are incorrectly powered in the presence of serially correlated errors, even with proper ex post inference. Our theoretical results enable us to correct this mismatch between ex ante and ex post assumptions on the error structure, and our serial-correlation-robust power calculation technique achieves the desired power in both simulated and real data. Ultimately, we provide researchers with both the theoretical insights and practical tools to design well-powered experiments in panel data settings.

We make three main contributions to the literature on experimental design in economics. First, we show that existing power calculation methods for panel data in economics, discussed in McKenzie (2012), fail in the presence of arbitrary serial correlation. We demonstrate this both analytically and via Monte Carlo using real and simulated data. Second, we derive a new expression for the variance of the difference-in-differences estimator under arbitrary serial correlation, which enables us to calibrate panel RCTs to the desired power. Finally, we address practical challenges involved in performing power calculations on panel data real experimental settings.

4. See Cameron and Miller (2015) for a practical guide to CRVE standard errors, which were first proposed by White (1984), and popularized by Arellano (1987). 
The paper proceeds as follows. Section 2 provides background on power calculations. Section 3 presents analytical power calculations expressions for panel data, and demonstrates their importance in Monte Carlo simulations with serially correlated errors. Section 4 applies these results to real experimental data. Section 5 discusses a few alternative estimation strategies and extensions. Section 6 discusses issues in designing panel RCTs and conducting power calculations in practice. Section 7 concludes.

\section{Background}

Randomized controlled trials allow researchers to overcome the fundamental challenge of causal inference highlighted by Rubin (1974): we can never observe the outcome for the same unit $i$ under treatment and control conditions simultaneously. RCTs solve this problem in expectation, by randomly assigning treatment to a subset of a population. Comparing the average outcomes of treated and untreated ("control") populations, researchers can identify the average causal effect of treatment. RCTs, and quasi-experimental research designs that attempt to mimic them, have been an important part of the ongoing empirical "credibility revolution" in economics (Angrist and Pischke (2010)).

Designing randomized experiments is challenging, in part because researchers have many degrees of freedom when doing so. They must choose a study location and sampling frame, select a sample size, implement an intervention, and collect data, all subject to partnerships with implementing agencies and to financial constraints. The choice of sample size is of particular importance, as it forces researchers to balance implementation costs and statistical power. Because recruitment and implementation of subjects is costly, an experiment should avoid excessively large samples. At the same time, an experiment that is too small will not be able to statistically distinguish between true and false null hypotheses.

A power calculation computes the smallest effect size that an experiment, with a given sample size and experimental design, will statistically be able to detect. The most general power calculation equation is:

$$
M D E=\left(t_{1-\kappa}^{d}+t_{\alpha / 2}^{d}\right) \sqrt{\operatorname{Var}(\hat{\tau} \mid \mathbf{X})}
$$

where $\operatorname{Var}(\hat{\tau} \mid \mathbf{X})$ is the exact finite sample variance of the treatment effect estimator, conditional

on independent variables $\mathbf{X} ; t_{\alpha / 2}^{d}$ is the critical value of a $t$ distribution with $d$ degrees of freedom 
associated with the probability of a Type I error, $\alpha$, in a two-sided test against a null hypothesis of $\tau=0$; and $t_{1-\kappa}^{d}$ is the critical value associated with the probability of correctly rejecting a false null, $\kappa .^{5}$ These parameters determine the minimum detectable effect $(M D E)$, the smallest value $|\tau|>0$ for which the experiment will (correctly) reject the null $\tau=0$ with probability $\kappa$ at the significance level $\alpha$.

Figure 1 illustrates these concepts graphically. The black curve represents the distribution of $\hat{\tau}$ if the null hypothesis is true, and the blue curve represents the distribution of $\hat{\tau}$ if the null hypothesis is false, where $\tau$ is instead equal to some value $\tau \neq 0$. Note that the variances of these distributions decrease with the sample size of the experiment. The dashed gray line is the critical value $t_{\alpha / 2}^{d}$. The shaded gray areas represent the likelihood that the researcher will reject a true null, and the blue-shaded area represents the statistical power of the test, or the probability that the experiment will correctly reject a false null. Figure 1 displays the case in which $\tau=M D E$, the minimum detectable effect size calibrated to the variance of $\hat{\tau}$, Type I error tolerance $\alpha$, and desired power $\kappa$.

While $\alpha$ and $\kappa$ are conventionally set to 0.05 and 0.80 , respectively, researcher choices govern the estimator $\hat{\tau}$. The variance of $\hat{\tau}$ depends jointly on the experimental design, the sample size, the model used to estimate $\hat{\tau}$, and the underlying properties of the data. To illustrate this, we first follow Bloom (1995) and Duflo, Glennerster, and Kremer (2007) in considering perhaps the simplest experimental design: a cross-sectional RCT. In this setup, $J$ units are randomly assigned a treatment status $D_{i}$, with proportion $P$ in treatment $\left(D_{i}=1\right)$ and proportion $(1-P)$ in control $\left(D_{i}=0\right)$. We make standard assumptions for randomized trials:

Assumption 1 (Data generating process). The data are generated according to the following model:

$$
Y_{i}=\beta+\tau D_{i}+\varepsilon_{i}
$$

where $\varepsilon_{i}$ is distributed i.i.d. $\mathcal{N}\left(0, \sigma_{\varepsilon}^{2}\right)$; and the treatment effect, $\tau$, is homogeneous across all units.

Assumption 2 (Strict exogeneity). $\mathrm{E}\left[\varepsilon_{i} \mid \mathbf{X}\right]=0$, where $\mathbf{X}=\left[\begin{array}{ll}\beta & D\end{array}\right]$. In practice, this follows from random assignment of $D_{i}$.

5. For one-sided tests, $t_{\alpha / 2}^{d}$ can be replaced with $t_{\alpha}^{d} .1-\kappa$ gives the probability of a false rejection, or a Type II error. The degrees of freedom, $d$, will depend on the dimensions of $\mathbf{X}$ and the treatment effect estimator in question. 
Figure 1: Hypothesis testing framework

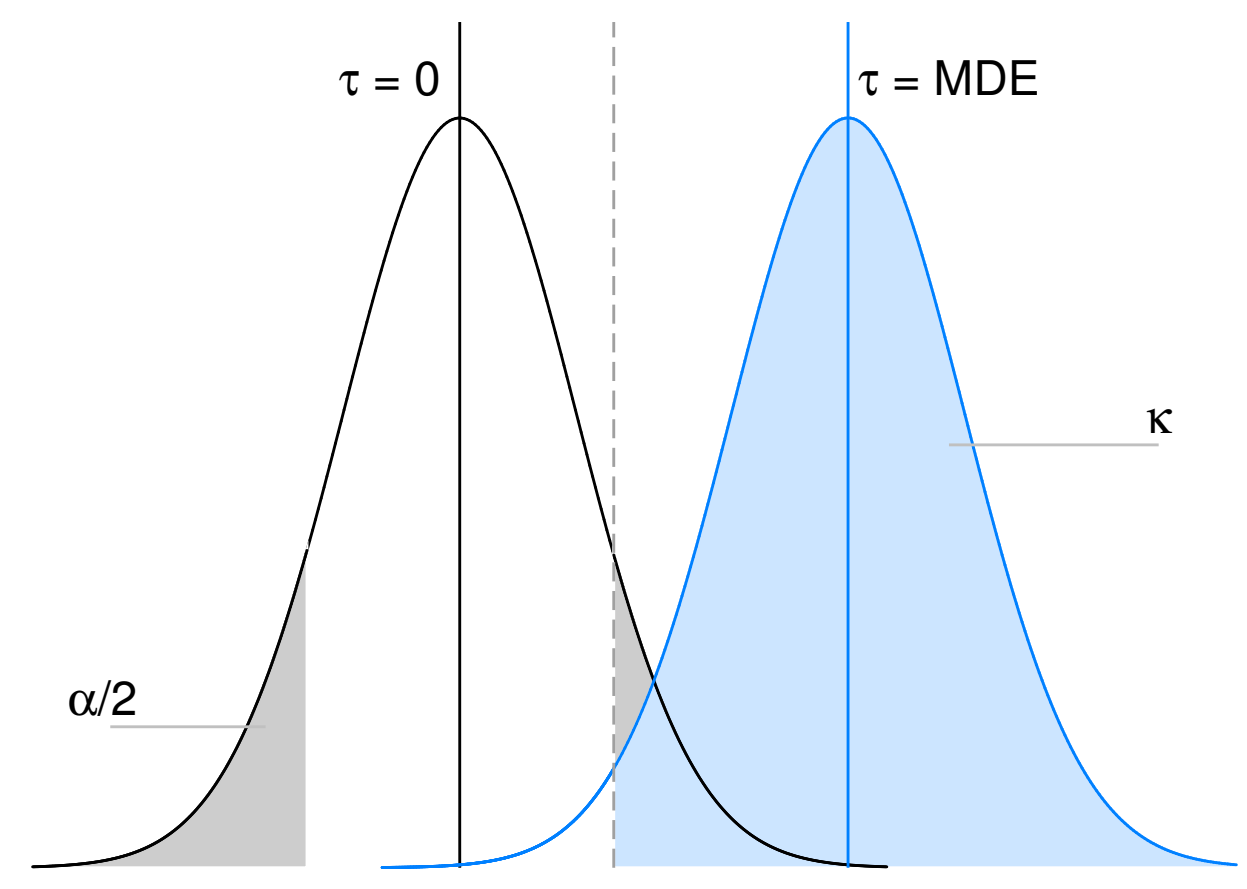

Notes: This figure displays the theoretical underpinnings of statistical power calculations. The black curve represents the distribution of the treatment effect estimator $\hat{\tau}$ under the null hypothesis of a zero effect. For a chosen significance level $\alpha$, we will reject this null if $\hat{\tau}$ lies above (below) the $1-\alpha / 2(\alpha / 2)$ percentile of the distribution. The gray-shaded area represents the likelihood of a Type I error. The blue curve is the distribution of $\hat{\tau}$ under the hypothesis that $\tau$ is equal to some other value, where this value is the minimum detectable effect $(M D E)$ that yields a statistical power of $\kappa$. Given that $\tau=M D E$ and given the sample size $J$, the shaded blue area is the power of this test. The unshaded area to the left of the critical value (the dashed gray line) and under the blue distribution represents the likelihood of committing a Type II error.

Define the OLS estimator of $\tau$ to be $\hat{\tau}=\left(\mathbf{X}^{\prime} \mathbf{X}\right)^{-1} \mathbf{X}^{\prime} Y$. Under Assumptions 1,2$]^{6}$

$$
\begin{aligned}
\mathrm{E}[\hat{\tau}-\tau \mid \mathbf{X}] & =0 \\
\operatorname{Var}(\hat{\tau} \mid \mathbf{X}) & =\frac{\sigma_{\varepsilon}^{2}}{P(1-P) J} \\
M D E & =\left(t_{1-\kappa}^{J-2}+t_{\alpha / 2}^{J-2}\right) \sqrt{\frac{\sigma_{\varepsilon}^{2}}{P(1-P) J}}
\end{aligned}
$$

Intuitively, the $M D E$ decreases with sample size $J$, increases with error variance, $\sigma_{\varepsilon}^{2}$, and is minimized at $P=0.5$. Given $\alpha$ and $\kappa$, larger experiments with less noisy data can statistically reject the null of zero for smaller true treatment effects.

6. See Appendix A.1.1 for a full derivation of the variance of $\hat{\tau}$ in this model. 
Researchers are not limited to this simple cross-sectional RCT design, however. Alternative designs and estimators may yield similar MDEs at lower cost. McKenzie (2012) highlights the possibility of using multiple waves of data in conjunction with a difference-in-difference (DD) estimator to decrease the number of units required to achieve a given $M D E$. In this model, $P$ proportion of the $J$ units are again randomized into treatment. The researcher collects the outcome $Y_{i t}$ for each unit $i$, across $m$ pre-treatment time periods and $r$ post-treatment time periods. For units in the treatment group, $D_{i t}=0$ in pre-treatment periods and $D_{i t}=1$ in post-treatment periods; for units in the control group, $D_{i t}=0$ in all $(m+r)$ periods.

Assumption 3 (Data generating process). The data are generated according to the following model:

$$
Y_{i t}=\beta+\tau D_{i t}+v_{i}+\delta_{t}+\omega_{i t}
$$

where $v_{i}$ is a unit-specific disturbance distributed i.i.d. $\mathcal{N}\left(0, \sigma_{v}^{2}\right) ; \delta_{t}$ is a time-specific disturbance distributed i.i.d. $\mathcal{N}\left(0, \sigma_{\delta}^{2}\right) ; \omega_{i t}$ is an idiosyncratic error term distributed i.i.d. $\mathcal{N}\left(0, \sigma_{\omega}^{2}\right)$; and the treatment effect, $\tau$, is homogeneous across all units and all time periods..$^{7}$

Assumption 4 (Strict exogeneity). $\mathrm{E}\left[\omega_{i t} \mid \mathbf{X}\right]=0$, where $\mathbf{X}$ is a full rank matrix of regressors, including a constant, the treatment indicator $D, J-1$ unit fixed effects, and $(m+r)-1$ time fixed effects. This again follows from random assignment of $D_{i t}$.

Assumption 5 (Balanced panel). The number of pre-treatment observations, $m$, and post-treatment observations, $r$, is the same for each unit, and all units are observed in every time period.

The OLS estimator of $\tau$ with unit and time fixed effects is $\widehat{\tau}=\left(\ddot{D} \ddot{D}^{-1} \ddot{D}^{\prime} \ddot{Y}\right.$, where:

$$
\begin{aligned}
& \ddot{Y}_{i t}=Y_{i t}-\frac{1}{m+r} \sum_{t} Y_{i t}-\frac{1}{J} \sum_{i} Y_{i t}+\frac{1}{J(m+r)} \sum_{i} \sum_{t} Y_{i t} \\
& \ddot{D}_{i t}=D_{i t}-\frac{1}{m+r} \sum_{t} D_{i t}-\frac{1}{J} \sum_{i} D_{i t}+\frac{1}{J(m+r)} \sum_{i} \sum_{t} D_{i t}
\end{aligned}
$$

7. This is the standard model used in panel RCTs. We assume here that these models are properly specified.

8. Under the assumption that the researcher knows the true model, random effects is more efficient than fixed effects. In practice, however, this is rarely the case, and researchers use fixed effects instead of random effects. Hence, we consider the fixed effects estimator here. 
Under Assumptions 35 .

$$
\begin{aligned}
\mathrm{E}[\hat{\tau}-\tau \mid \mathbf{X}] & =0 \\
\operatorname{Var}(\hat{\tau} \mid \mathbf{X}) & =\left(\frac{\sigma_{\omega}^{2}}{P(1-P) J}\right)\left(\frac{m+r}{m r}\right) \\
M D E & =\left(t_{1-\kappa}^{J}+t_{\alpha / 2}^{J}\right) \sqrt{\left(\frac{\sigma_{\omega}^{2}}{P(1-P) J}\right)\left(\frac{m+r}{m r}\right)}
\end{aligned}
$$

This is the power calculation equation originally derived by Frison and Pocock (1992) (henceforth FP)..$^{9}$ The experiment's $M D E$ decreases symmetrically in $m$ and $r$, because, holding the error variance constant, longer panels decrease the variance of the DD estimator. Note that researchers can potentially trade off $J$ for $m$ and/or $r$ to decrease both $M D E$ and implementation costs.

Importantly, $\sigma_{\omega}^{2} \leq \sigma_{\varepsilon}^{2}$ by construction, since $\omega_{i t}$ represents only the idiosyncratic component of the error term. Empirically, the inclusion of fixed effects reduces the error variance to the extent that underlying within-unit and within-time correlations explain $Y_{i t}$. To see this, we can rewrite Equation (3) in terms of $\sigma_{\varepsilon}^{2}$. Let $\rho_{v}$ and $\rho_{\delta}$ denote the proportion of the composite variance $\sigma_{\varepsilon}^{2}$ contributed by $\sigma_{v}^{2}$ and $\sigma_{\delta}^{2}$, respectively:

$$
\rho_{v} \equiv \frac{\sigma_{v}^{2}}{\sigma_{v}^{2}+\sigma_{\delta}^{2}+\sigma_{\omega}^{2}}=\frac{\sigma_{v}^{2}}{\sigma_{\varepsilon}^{2}} \quad \rho_{\delta} \equiv \frac{\sigma_{\delta}^{2}}{\sigma_{v}^{2}+\sigma_{\delta}^{2}+\sigma_{\omega}^{2}}=\frac{\sigma_{\delta}^{2}}{\sigma_{\varepsilon}^{2}}
$$

Then, (3) can be rewritten as:

$$
M D E=\left(t_{1-\kappa}^{J}+t_{\alpha / 2}^{J}\right) \sqrt{\left(\frac{\sigma_{\varepsilon}^{2}}{P(1-P) J}\right)\left(\frac{m+r}{m r}\right)\left(1-\rho_{v}-\rho_{\delta}\right)}
$$

In this formula, larger unit-level intracluster correlations (i.e., $\rho_{v}$ closer to 1 ) or stronger temporal shocks (i.e., $\rho_{\delta}$ closer to 1) yield smaller MDEs.10 Notice that, although Equation (4) includes intracluster correlation coefficient terms, the idiosyncratic component of the error term $\left(\omega_{i t}\right)$ is still assumed to be i.i.d. This highlights an important point: accounting for intracluster correlation is

9. See Appendix A.2.1 for a full derivation. Here, we use critical values with $J$ degrees of freedom, which is consistent with the assumptions of the CRVE with $J$ clusters. By contrast, the OLS variance estimator would use $J(m+r)-(J+m+r)$ degrees of freedom.

10. Replacing $\rho_{\delta}=0, P=0.5$, and $J=2 n$, Equation 4 is equivalent to the power calculation formula for difference-in-differences derived by FP and discussed in McKenzie (2012). See Appendix A.2.1 for complete derivations. Equation (3) is not identical to the model in FP or McKenzie (2012), because these authors assume that the time disturbance $\delta_{t}$ is deterministic and has no variance. Our model allows for $\sigma_{\delta}^{2}>0$, in keeping with assumptions economists typically make about data generating processes. Hence, Equation (3) represents a more general version of the FP formula. 
not the same as allowing for arbitrary serial correlation. Indeed, Bertrand, Duflo, and Mullainathan (2004) (henceforth BDM) demonstrate that panel data are likely to exhibit serial correlation within units, meaning that the assumption of i.i.d. errors is unlikely to hold in practice.

\section{Theory}

\subsection{Correlated errors in panel models}

Power calculation formulas such as the standard cross-sectional model (Equation (2p) and the FP model (Equation (3)) assume that the error structure in data results from an i.i.d. process. Real data rarely exhibit i.i.d. errors, and researchers frequently apply the CRVE to allow for correlated errors. In cases where treatment is randomly assigned across cross-sectional units, however, the OLS variance estimator is an unbiased estimator of the true variance, even in the presence of cross-sectional error correlations:

Lemma 1. In a cross-sectional model with random assignment to treatment, $\frac{\sigma_{\varepsilon}^{2}}{P(1-P) J}$ is an unbiased estimator of $\operatorname{Var}(\hat{\tau} \mid \mathbf{X})$ even if $\mathrm{E}\left[\varepsilon_{i} \varepsilon_{j} \mid \mathbf{X}\right] \neq 0$ for some $i \neq j$. See Appendix A.3 for a proof ${ }^{11}$

This means that in single-wave RCTs, researchers need not adjust standard errors to account for correlation across experimental units.

Cross-sectional randomization does not obviate the need to account for serially correlated errors in panel datasets. When an experimenter collects data from the same cross-sectional units over multiple time periods, each unit's error terms are likely correlated across time.12 In most DD research designs, once treatment begins, it persists for the remainder of the study, so both a unit's error terms and its treatment status are serially correlated. Hence, researchers still need to account for serial correlation when randomizing treatment at the unit level.13 BDM demonstrate

11. Campbell (1977) provides the first version of this proof, which is cited by Moulton (1986), and which imposes a grouped error structure. In Appendix A.3 we provide a proof which allows for arbitrary cross-sectional error dependence. Athey and Imbens (2016a, 2016b) still recommend using Eicker-Huber-White standard errors in this case, to allow for heteroskedasticity. We do not know of a paper that discusses power calculations in the presence of heteroskedastic disturbances.

12. This is true even in a model with unit and time period fixed effects. These fixed effects control for the average outcome of each unit across all time periods, and the average outcome across all units in each time period. However, if each unit's demeaned outcome realizations evolve non-independently across time, then the resulting "idiosyncratic" error terms (i.e., $\omega_{i t}$ in Equation (3)) will exhibit some form of correlation that violates the i.i.d. assumption.

13. As with single-wave RCTs, cross-sectional randomization in panel RCTs eliminates the need to adjust for crosssectional correlations. Randomizing the timing and duration of treatment within treated units would make the OLS variance estimator unbiased, but would be logistically prohibitive in most settings. 
that serial correlation in DD designs can severely bias conventional standard errors towards zero. This means that failing to account for serially correlated errors can lead to high Type I error rates and substantial over-rejection of true null hypotheses.

Given that a panel DD analysis should account for potential serial correlation ex post, what does this imply for the ex ante statistical power of such an experiment? BDM find that while applying the CRVE on a serially correlated panel dataset can reduce the Type I error rate to the desired level, this has the effect of increasing the Type II error rate. In other words, correctly accounting for serial correlation will tend to inflate standard errors, which in turn will reduce the rejection rates of both false and true null hypotheses. If a researcher designs a DD experiment using the FP power calculation formula, and then applies the CRVE ex post, this suggests that her experiment will likely be underpowered.

\subsection{Power calculations with serial correlation}

We derive a more general version of the FP DD power calculation formula in order to accommodate the non-i.i.d. error structures, including arbitrary correlations within cross-sectional units over time, which are present in real-world data 14 Just as in the FP model, there are $J$ units, $P$ proportion of which are randomized into treatment. The researcher again collects outcome data $Y_{i t}$ for each unit $i$, across $m$ pre-treatment time periods and $r$ post-treatment time periods. For treated units, $D_{i t}=0$ in pre-treatment periods, and $D_{i t}=1$ in post-treatment periods; for control units, $D_{i t}=0$ in all periods.

Assumption 6 (Data generating process). The data are generated according to the following model:

$$
Y_{i t}=\beta+\tau D_{i t}+v_{i}+\delta_{t}+\omega_{i t}
$$

where $v_{i}$ is a unit-specific disturbance distributed i.i.d. $\mathcal{N}\left(0, \sigma_{v}^{2}\right) ; \delta_{t}$ is a time-specific disturbance distributed i.i.d. $\mathcal{N}\left(0, \sigma_{\delta}^{2}\right) ; \omega_{i t}$ is an idiosyncratic error term distributed (not necessarily i.i.d.) $\mathcal{N}\left(0, \sigma_{\omega}^{2}\right)$; and the treatment effect, $\tau$, is homogeneous across all units and all time periods.

14. We do not consider cross-sectional correlation, because we consider a treatment that is randomized at the unit level. For a full version of this model incorporating both arbitrary serial and cross-sectional correlations, see Appendix A.2.3. 
Assumption 7 (Strict exogeneity). $\mathrm{E}\left[\omega_{i t} \mid \mathbf{X}\right]=0$, where $\mathbf{X}$ is a full rank matrix of regressors, including a constant, the treatment indicator $D, J-1$ unit fixed effects, and $(m+r)-1$ time fixed effects. This again follows from random assignment of $D_{i t}$.

Assumption 8 (Balanced panel). The number of pre-treatment observations, $m$, and post-treatment observations, $r$, is the same for each unit, and all units are observed in every time period.

Assumption 9 (Independence across units). $\mathrm{E}\left[\omega_{i t} \omega_{j s} \mid \mathbf{X}\right]=0, \forall i \neq j, \forall t, s$.

Assumption 10 (Symmetric covariance structures). Define:

$$
\begin{aligned}
\psi_{T}^{B} & \equiv \frac{2}{P J m(m-1)} \sum_{i=1}^{P J} \sum_{t=-m+1}^{-1} \sum_{s=t+1}^{0} \operatorname{Cov}\left(\omega_{i t}, \omega_{i s} \mid \mathbf{X}\right) \\
\psi_{T}^{A} & \equiv \frac{2}{P J r(r-1)} \sum_{i=1}^{P J} \sum_{t=1}^{r-1} \sum_{s=t+1}^{r} \operatorname{Cov}\left(\omega_{i t}, \omega_{i s} \mid \mathbf{X}\right) \\
\psi_{T}^{X} & \equiv \frac{1}{P J m r} \sum_{i=1}^{P J} \sum_{t=-m+1}^{0} \sum_{s=1}^{r} \operatorname{Cov}\left(\omega_{i t}, \omega_{i s} \mid \mathbf{X}\right)
\end{aligned}
$$

to be the average pre-treatment, post-treatment, and across-period covariance between different error terms of the same treated unit, respectively. Define $\psi_{C}^{B}, \psi_{C}^{A}$, and $\psi_{C}^{X}$ analogously, where we consider the $(1-P) J$ control units instead of the PJ treated units. Using these definitions, assume that $\psi^{B}=\psi_{T}^{B}=\psi_{C}^{B} ; \psi^{A}=\psi_{T}^{A}=\psi_{C}^{A} ;$ and $\psi^{X}=\psi_{T}^{X}=\psi_{C}^{X} 15$

The OLS estimator with unit and time fixed effects remains $\widehat{\tau}=\left(\ddot{D}^{\prime} \ddot{D}\right)^{-1} \ddot{D}^{\prime} \ddot{Y}$ and again, $\mathrm{E}[\hat{\tau}-\tau \mid$ $\mathbf{X}]=0$. However, Assumptions 60 extend FP to a more general power calculation formula that incorporates arbitrary within-unit correlations: ${ }^{16}$

$$
\begin{aligned}
\operatorname{Var}(\hat{\tau} \mid \mathbf{X}) & =\left(\frac{1}{P(1-P) J}\right)\left[\left(\frac{m+r}{m r}\right) \sigma_{\omega}^{2}+\left(\frac{m-1}{m}\right) \psi^{B}+\left(\frac{r-1}{r}\right) \psi^{A}-2 \psi^{X}\right] \\
M D E & =\left(t_{1-\kappa}^{J}+t_{\alpha / 2}^{J}\right) \sqrt{\left(\frac{1}{P(1-P) J}\right)\left[\left(\frac{m+r}{m r}\right) \sigma_{\omega}^{2}+\left(\frac{m-1}{m}\right) \psi^{B}+\left(\frac{r-1}{r}\right) \psi^{A}-2 \psi^{X}\right]}
\end{aligned}
$$

15. We choose the letters "B" to indicate the Before-treatment period, and " $\mathrm{A}$ " to indicate the After-treatment period. We index the $m$ pre-treatment periods $\{-m+1, \ldots, 0\}$, and the $r$ post-treatment periods $\{1, \ldots, r\}$. In a randomized setting, $\mathrm{E}\left[\psi_{T}^{B}\right]=\mathrm{E}\left[\psi_{C}^{B}\right], \mathrm{E}\left[\psi_{T}^{A}\right]=\mathrm{E}\left[\psi_{C}^{A}\right]$, and $\mathrm{E}\left[\psi_{T}^{X}\right]=\mathrm{E}\left[\psi_{C}^{X}\right]$, making this a reasonable assumption ex ante. However, it is possible for treatment to alter the covariance structure of treated units only.

16. We present the formal derivation of this formula in Appendix A.2.2. Note that if $m=1$ (or $r=1$ ), $\psi^{B}$ (or $\psi^{A}$ ) is not defined and is multiplied by 0 in Equation (5). 
Throughout the remainder of the paper, we refer to Equation (5) as the "serial-correlation-robust" (SCR) power calculation formula. Note that under cross-sectional randomization, this expression for the variance of $\hat{\tau}$ still holds in expectation, even in the presence of within-period error correlations across units:

Lemma 2. In a panel difference-in-differences model with treatment randomly assigned at the unit level, $\left(\frac{1}{P(1-P) J}\right)\left[\left(\frac{m+r}{m r}\right) \sigma_{\omega}^{2}+\left(\frac{m-1}{m}\right) \psi^{B}+\left(\frac{r-1}{r}\right) \psi^{A}-2 \psi^{X}\right]$ is an unbiased estimator of $\operatorname{Var}(\hat{\tau} \mid \mathbf{X})$, even in the presence of arbitrary within-period cross-sectional correlations. See Appendix A.3 for a proof, and see Appendix A.2.3 for a more general model that relaxes Assumptions 9 [10.

To illustrate the difference between the FP and SCR models, consider two cross-sectional units (indexed $\{i, j\}$ ) and four time periods (indexed $\{0,1,2,3\}$ ). The vector of errors, $\vec{\omega}$, and the corresponding variance-covariance matrix, $\Omega$, can be represented as follows: ${ }^{17}$

$$
\vec{\omega}=\left[\begin{array}{c}
\omega_{i 0} \\
\omega_{i 1} \\
\omega_{i 2} \\
\omega_{i 3} \\
\omega_{j 0} \\
\omega_{j 1} \\
\omega_{j 2} \\
\omega_{j 3}
\end{array}\right] \quad \Omega=\left[\begin{array}{cccccccc}
\sigma_{i 0}^{2} \\
\sigma_{i 0, i 1} & \sigma_{i 1}^{2} & & & & & & \\
\sigma_{i 0, i 2} & \sigma_{i 1, i 2} & \sigma_{i 2}^{2} & & & & \\
\sigma_{i 0, i 3} & \sigma_{i 1, i 3} & \sigma_{i 2, i 3} & \sigma_{i 3}^{2} & & & \\
& & & & \sigma_{j 0}^{2} & & & \\
& & & & & & \\
& & & \sigma_{j 0, j 1} & \sigma_{j 1}^{2} & & \\
& & & \sigma_{j 0, j 2} & \sigma_{j 1, j 2} & \sigma_{j 2}^{2} & \\
& & & \sigma_{j 0, j 3} & \sigma_{j 1, j 3} & \sigma_{j 2, j 3} & \sigma_{j 3}^{2}
\end{array}\right]
$$

Serial correlation within each unit is represented by the (potentially non-zero) covariance terms $\sigma_{i t, i s}$ and $\sigma_{j t, j s}$, for all $t \neq s$. In contrast, the FP model assumes that these off-diagonal covariance elements are all zero.

The magnitudes of these off-diagonal covariance terms directly affect the variance of the DD estimator. The three $\psi$ terms defined above, along with the error variance and experimental design parameters, are sufficient to fully characterize the true variance of the treatment effect estimator in this model. To fix ideas, using the four-period model above, and supposing treatment is administered

17. We show only the lower diagonal of the variance-covariance matrix because the full matrix is symmetric. Note further that we do not show the cross-unit covariance terms for notational convenience, as these terms are assumed to be zero. 
beginning at $t=2$, these three covariance parameters are:

$$
\begin{aligned}
\psi^{B} & =\frac{\sigma_{i 0, i 1}+\sigma_{j 0, j 1}}{2} \\
\psi^{A} & =\frac{\sigma_{i 2, i 3}+\sigma_{j 2, j 3}}{2} \\
\psi^{X} & =\frac{\sigma_{i 0, i 2}+\sigma_{i 1, i 2}+\sigma_{i 0, i 3}+\sigma_{i 1, i 3}+\sigma_{j 0, j 2}+\sigma_{j 1, j 2}+\sigma_{j 0, j 3}+\sigma_{j 1, j 3}}{8}
\end{aligned}
$$

Alternatively, if treatment is administered beginning at $t=1$, these covariance terms become:

$$
\begin{aligned}
\psi^{B} & =\text { (not defined for only } 1 \text { pre-treatment period }) \\
\psi^{A} & =\frac{\sigma_{i 1, i 2}+\sigma_{i 1, i 3}+\sigma_{i 2, i 3}+\sigma_{j 1, j 2}+\sigma_{j 1, j 3}+\sigma_{j 2, j 3}}{6} \\
\psi^{X} & =\frac{\sigma_{i 0, i 1}+\sigma_{i 0, i 2}+\sigma_{i 0, i 3}+\sigma_{j 0, j 1}+\sigma_{j 0, j 2}+\sigma_{j 0, j 3}}{6}
\end{aligned}
$$

The SCR power calculation formula above generalizes this structure to a model with $J$ units across $m$ pre-treatment periods and $r$ post-treatment periods. In this model, greater average covariance in the pre- or post-treatment periods $\left(\psi^{B}\right.$ or $\left.\psi^{A}\right)$ increases the $M D E$. Intuitively, as errors for treated and control units are more serially correlated, the benefits of collecting multiple waves of pre- and post-treatment data are eroded. However, cross-period covariance $\left(\psi^{X}\right)$ enters the MDE formula negatively. This highlights a key property of the DD estimator - because DD identifies the treatment effect off of differences between post- and pre-treatment outcomes, greater serial correlation between pre- and post-treatment observations makes differences caused by treatment easier to detect.

Assuming that the within-unit correlation structure does not vary systematically across time periods, positively correlated errors will imply positive $\psi^{B}, \psi^{A}$, and $\psi^{X}$. Because $\psi^{B}$ and $\psi^{A}$ enter the SCR power calculation formula positively, while $\psi^{X}$ enters negatively, serial correlation may either increase or decrease the $M D E$ relative to the i.i.d. case. Specifically, serial correlation will increase the $M D E$ if and only if:

$$
\left(\frac{m-1}{m}\right) \psi^{B}+\left(\frac{r-1}{r}\right) \psi^{A}>2 \psi^{X}
$$

This inequality is more likely to hold in longer panels, for two reasons. First, as the number of pre- and post-treatment periods increases, $\left(\frac{m-1}{m}\right)$ and $\left(\frac{r-1}{r}\right)$ approach one. Second, the covariance terms contributing to $\psi^{X}$ lie farther away from the diagonal of the variance-covariance matrix than 
the covariance terms contributing to $\psi^{B}$ and $\psi^{A}$. Because errors from non-adjacent time periods are likely to be less correlated than errors from adjacent time periods, and because the number of far-off-diagonal covariances increases relatively more quickly for $\psi^{X}$ as the panel becomes longer, $\psi^{X}$ is increasingly likely to be smaller than $\psi^{B}$ and $\psi^{A}$ in longer panels. Together, these two effects imply that for longer panels, the FP model is increasingly likely to yield underpowered experiments. At the same time, using FP with short panels is likely to yield overpowered experiments.

\subsection{Monte Carlo simulations}

If a randomized experiment relies on a power calculation that fails to account for serial correlation ex ante, its realized power may be different from the desired $\kappa$. To understand the extent to which this matters in practice, we conduct a series of Monte Carlo simulations comparing the FP model and the SCR model over a range of panel lengths and error correlations. We simulate three cases and compute the Type I error rate and the statistical power for each: (i) experiments that fail to account for serial correlation both ex ante and ex post; (ii) experiments that fail to account for serial correlation ex ante but apply the CRVE to account for serial correlation ex post; and (iii) experiments that both account for serial correlation ex ante and apply the CRVE ex post.

For each set of parameter values characterizing both a data generating process and an experimental design, we first calculate two treatment effect sizes: $\tau^{F P}$ equal to the $M D E$ from the FP

formula, and $\tau^{S C R}$ equal to the $M D E$ from our SCR formula. Second, we use these parameter values to create a panel dataset from the following data generating process:

$$
Y_{i t}=\beta+v_{i}+\delta_{t}+\omega_{i t}
$$

where $\omega_{i t}$ follows an $\operatorname{AR}(1)$ process:

$$
\omega_{i t}=\gamma \omega_{i(t-1)}+\xi_{i t}
$$

Third, we randomly assign treatment, with effect sizes $\tau^{F P}, \tau^{S C R}$, and $\tau^{0}=0$ at the unit level, to create three separate outcome variables. Fourth, we regress each of these outcome variables on their respective treatment indicators, unit fixed effects, and time fixed effects. Fifth, we compute both OLS standard errors and CRVE standard errors clustered at the unit level, for all three regressions. We repeat steps two through five 10,000 times for each set of parameters, calculating rejection rates 
of the null hypothesis $\tau=0$ across all simulations. For $\tau^{F P}$ and $\tau^{S C R}$, this rate represents the realized power of the experiment. For the placebo $\tau^{0}$, it represents the realized false rejection rate.

We test five levels of the $\operatorname{AR}(1)$ parameter: $\gamma \in\{0,0.3,0.5,0.7,0.9\}$. For each $\gamma$, we simulate symmetric panels with an equal number of pre-treatment and post-treatment periods, with panel lengths ranging from 2 periods $(m=r=1)$ to 40 periods $(m=r=20)$. We hold $J, P, \beta, \sigma_{v}^{2}, \sigma_{\delta}^{2}, \alpha$, and $\kappa$ fixed across all simulations, and we adjust the variance of the white noise term $\sigma_{\xi}^{2}$ such that every simulation has a fixed idiosyncratic variance $\sigma_{\omega}^{2}$. This allows $\gamma$ to govern the proportion of $\sigma_{\omega}^{2}$ that is serially correlated 18 The covariance terms $\psi^{B}, \psi^{A}$, and $\psi^{X}$ have closed-form expressions under the $\mathrm{AR}(1)$ structure, and we use these expressions to calculate $\tau^{S C R} \cdot{ }^{19}$ This causes $\tau^{S C R}$ to vary both with the degree of serial correlation and panel length, whereas $\tau^{F P}$ varies only with panel length.

Figure 2 displays the results of this exercise. The left column shows rejection rates under the FP formula using OLS standard errors, which assumes zero serial correlation both ex ante and ex post. The middle column shows rejection rates under the FP formula using CRVE standard errors, which accounts for serial correlation ex post only. The right column show rejection rates under our SCR formula using CRVE standard errors, which allows for serial correlation both ex ante and ex post. The top row plots realized power as a function of the number of pre/post-treatment periods, which should equal $\kappa=0.80$ in a properly designed experiment. The bottom row plots the corresponding realized false rejection rates, which should equal the desired $\alpha=0.05$. Only the SCR formula, in conjunction with CRVE standard errors, achieves the desired 0.80 and 0.05 across all panel lengths and $\operatorname{AR}(1)$ parameters.

The left column confirms the BDM result that failing to account for serial correlation leads to false rejection rates dramatically higher than $\alpha=0.05$. Even a modest serial correlation parameter of $\gamma=0.5$ yields a 20 percent probability of a Type I error, for panels with $m=r>5$. This underscores the fact that randomization cannot correct serial correlation in panel settings, and experiments that collect multiple waves of data from the same cross-sectional units should account for within-unit correlation over time. By contrast, the middle and right columns apply the CRVE and reject placebo effects at the desired rate of $\alpha=0.05$.

\footnotetext{
18. In an $\mathrm{AR}(1)$ model, the relationship between the variance of the $\mathrm{AR}(1)$ process and the variance of the white noise disturbance depends on $\gamma$, with $\sigma_{\omega}^{2}=\frac{\sigma_{\xi}^{2}}{1-\gamma^{2}}$.

19. We provide these formal derivations in Appendix B.1, along with further details on these Monte Carlo simulations. We also provide additional simulation results that separately vary $m$ and $r$ in Appendix C.
} 
Figure 2: Traditional methods result in improperly powered experiments in AR(1) data
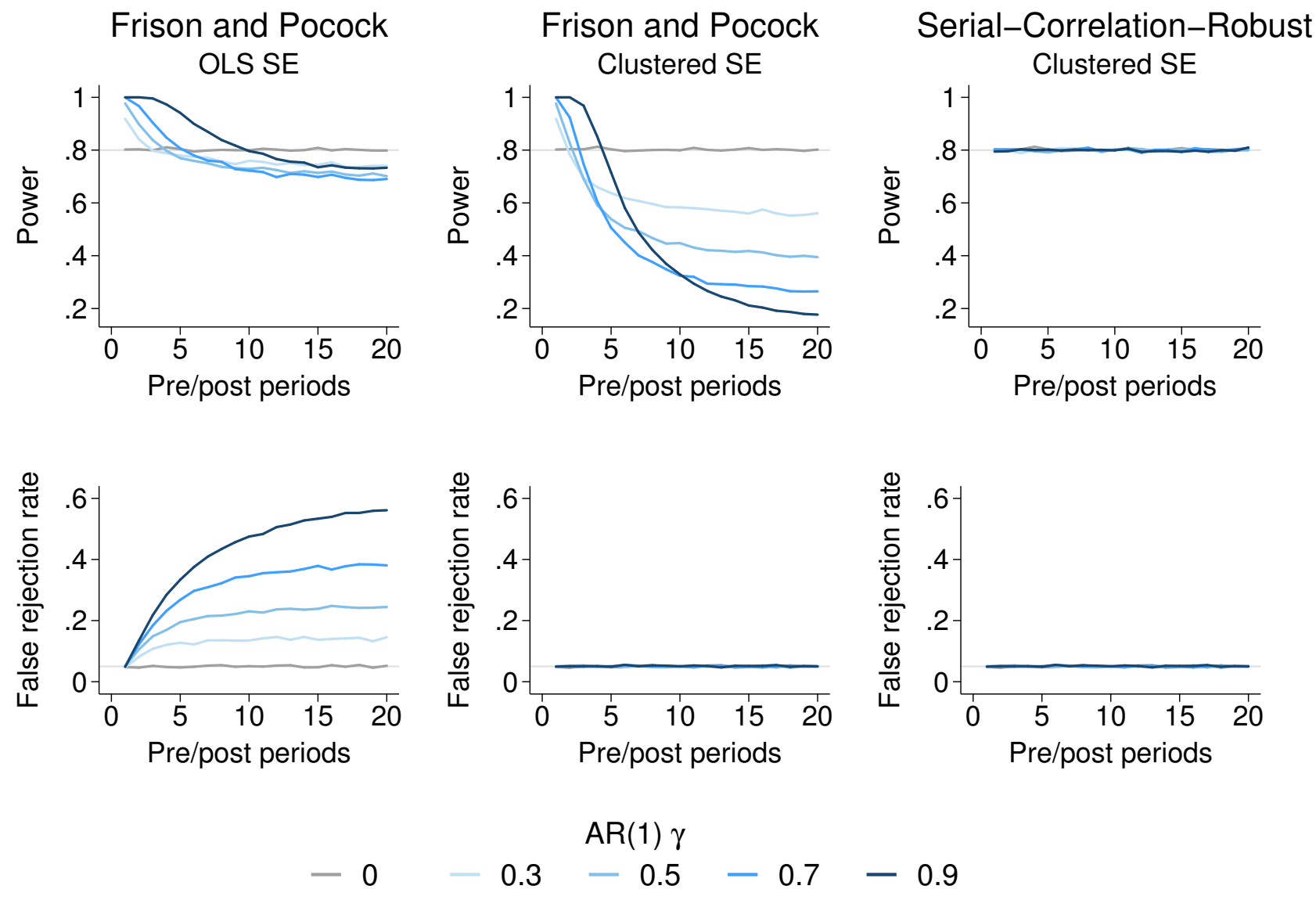

Notes: This figure displays power and rejection rates from performing power calculations with three different sets of assumptions on data generated with $\mathrm{AR}(1)$ processes with differing levels of serial correlation and differing panel lengths (ranging from 2, $m=r=1$, to $40, m=r=20$ ). In the left column, we apply the formula of Frison and Pocock (1992) (Equation (3)), and use OLS standard errors ex post, in line with the assumptions of this formula. In the middle column, we again apply calibrate power calculations using Frison and Pocock (1992)'s formula, but cluster standard errors ex post - which is inconsistent with the ex ante formula, but corrects for within-unit serial correlation following Bertrand, Duflo, and Mullainathan (2004). In the right column, we apply the serial-correlationrobust power calculation formula to account for non-i.i.d. errors ex ante, and we cluster standard errors at the individual level ex post. As expected, this third set of simulations achieves the desired 80 percent power and 5 percent false rejection rate.

The middle column shows how failing to account for serial correlation ex ante can yield dramatically overpowered or underpowered experiments. Particularly for longer panels with $m=r>5$, performing power calculations via Equation (3) may actually produce experiments with less than 50 percent power, even though researchers intended to achieve power of 80 percent (i.e., $\kappa=0.80$ ). For a relatively high serial correlation of $\gamma=0.7$, simulations based on the conventional power calculation formula yield power less than 32 percent, for $m=r>10$. This is broadly consistent with the BDM finding that applying the CRVE reduces statistical power, even though doing so 
achieves the desired Type I error rate. By contrast, the right column applies both the SCR power calculation formula and the CRVE, and these simulations achieve the desired power of $\kappa=0.80$ for each $\gamma$.

The middle column also highlights how failing to account for serial correlation ex ante may either increase or decrease statistical power, as shown in Equation (6). For shorter panels, using the FP formula instead of our SCR formula yields dramatically overpowered experiments. While this may seem counterintuitive, (6) is increasingly unlikely to hold as $m$ and $r$ decrease to 1 . In the extreme case where $m=r=1, \psi^{B}$ and $\psi^{A}$ do not enter, and the only covariance term in the SCR formula is $\psi^{X}$, which enters negatively. These simulations reveal that just as higher $\gamma$ yields more dramatically underpowered experiments for longer panels, higher $\gamma$ yields more dramatically overpowered experiments for shorter panels.20

These results are striking. For even a modest degree of serial correlation, applying the FP power calculation formula will not yield experiments of the desired statistical power. By contrast, the SCR formula achieves the desired 80 percent power for all panel lengths and AR(1) parameters. While $\mathrm{AR}(1)$ is a relatively simple correlation structure, it serves as a reasonable first-approximation for more complex forms of serial correlation. This exercise suggests that, in theory, researchers must account for serial correlation in experimental design. Given that real-world panel datasets exhibit enough serial correlation to produce high Type I error rates, it stands to reason that such serial correlation can similarly impact the statistical power of experiments if not accounted for ex ante.

\section{Applications to real-world data}

\subsection{Bloom et al. (2015) data}

In order to understand whether the differences in power demonstrated in the Monte Carlo simulations above are meaningful in practice, we conduct an analogous simulation exercise using a real dataset from an experiment in a developing-country setting. We use data from Bloom et al. (2015), in which Chinese call center employees were randomly assigned to work either from home or from the office for a nine-month period. The authors estimate the following equation to derive the central

\footnotetext{
20. Intuitively, serial correlation has two opposite effects on the statistical power of a DD estimator. It decreases power by reducing the effective number of observations for each cross-sectional unit, and it increases power by increasing the signal in estimating treatment effects off of a post-pre difference. In shorter panels, this second effect tends to dominate. See Appendix C for additional short-panel results.
} 
result, which is found in Table 2 in the original paper:

$$
\text { Performance }_{i t}=\alpha \text { Treat }_{i} \times \text { Experiment }_{t}+\beta_{t}+\gamma_{i}+\varepsilon_{i t}
$$

This is a standard DD estimating equation with fixed effects for individual $i$ and week $t$. The paper's dataset consists of weekly performance measures for the 249 workers enrolled in the experiment between January 2010 and August 2011. We perform simulations using data from the pre-treatment period only, to avoid contamination from the Bloom et al. (2015) intervention. We keep only those individuals who have non-missing performance data for the entire pre-treatment period, leaving us with a balanced panel of 79 individuals over 48 pre-treatment weeks. ${ }^{21}$ Table 1 provides summary statistics, including the $\operatorname{AR}(1) \hat{\gamma}$ parameter recovered by estimating Equation (8) on residuals from this dataset. Our estimate of $\hat{\gamma}=0.233$ is highly statistically significant, which indicates that these worker performance data exhibit weak serial correlation.

Table 1: Summary statistics - Bloom et al. (2015)

\begin{tabular}{cccccccc}
\hline \hline Mean & Std. Dev. & Min & Max & $\operatorname{AR}(1) \hat{\gamma}$ & Individuals & Periods & Observations \\
\hline 0.153 & 0.943 & -2.766 & 3.665 & 0.233 & 79 & 48 & 3,792 \\
\hline
\end{tabular}

Notes: This table shows summary statistics for worker productivity in the Bloom et al. (2015) data. The data are weekly job performance $z$-scores, constructed by taking the average of normalized performance measures, where each measure is standardized to have a mean of 0 and standard deviation of 1 across the sample. Our sample consists only of individuals that had no missing observations throughout the entire pre-treatment period, January 1, 2010 through November 28, 2010. We compute $\hat{\gamma}$ by estimating Equation (8) on residuals from this dataset. In doing so, we cluster standard errors at the individual level. The $95 \%$ confidence interval is $[0.165,0.300]$. For more details on the standardized job performance measures and the actual experimental design, see Bloom et al. (2015).

We perform Monte Carlo simulations on this dataset which are analogous to those presented above. We subset consecutive periods of the Bloom et al. (2015) dataset to create panels ranging in length from 2 periods $(m=r=1)$ to 40 periods $(m=r=20)$. For each panel length, we randomly assign three treatment effect sizes, $\tau^{F P}, \tau^{A R(1)}$, and $\tau^{S C R}$, at the individual level and estimate Equation (9) separately for each treatment effect size. We calibrate $\tau^{F P}$ using the FP formula, assuming zero serial correlation and estimating $\sigma_{\hat{\omega}}^{2}$ using residuals from these data. We calibrate $\tau^{A R(1)}$ using the SCR formula, deriving the $\psi$ parameters analytically by assuming an $\operatorname{AR}(1)$ error

21. This leaves us with a very different sample from Bloom et al. 2015). Our purpose with this exercise is not to comment on the statistical power of the original paper, but rather to investigate the importance of accounting for serial correlation ex ante in real experimental data. 
structure with $\gamma=0.233$. We calibrate $\tau^{S C R}$ using the SCR formula, where we non-parametrically estimate $\psi_{\hat{\omega}}^{B}, \psi_{\hat{\omega}}^{A}$, and $\psi_{\hat{\omega}}^{X}$ from residuals 22

Figure 3 reports the results of this exercise, demonstrating that only the SCR formula achieves the desired statistical power in the Bloom et al. (2015) data. In contrast, failing to account for serial correlation leads to experiments that deviate dramatically from the desired 80 percent power, even in the presence of relatively weak serial correlation. For an experiment with 12 pre/post-treatment periods, applying the FP formula with $\kappa=0.80$ yields an experiment with only 35 percent power.

Figure 3: Power simulations for Bloom et al. (2015) data

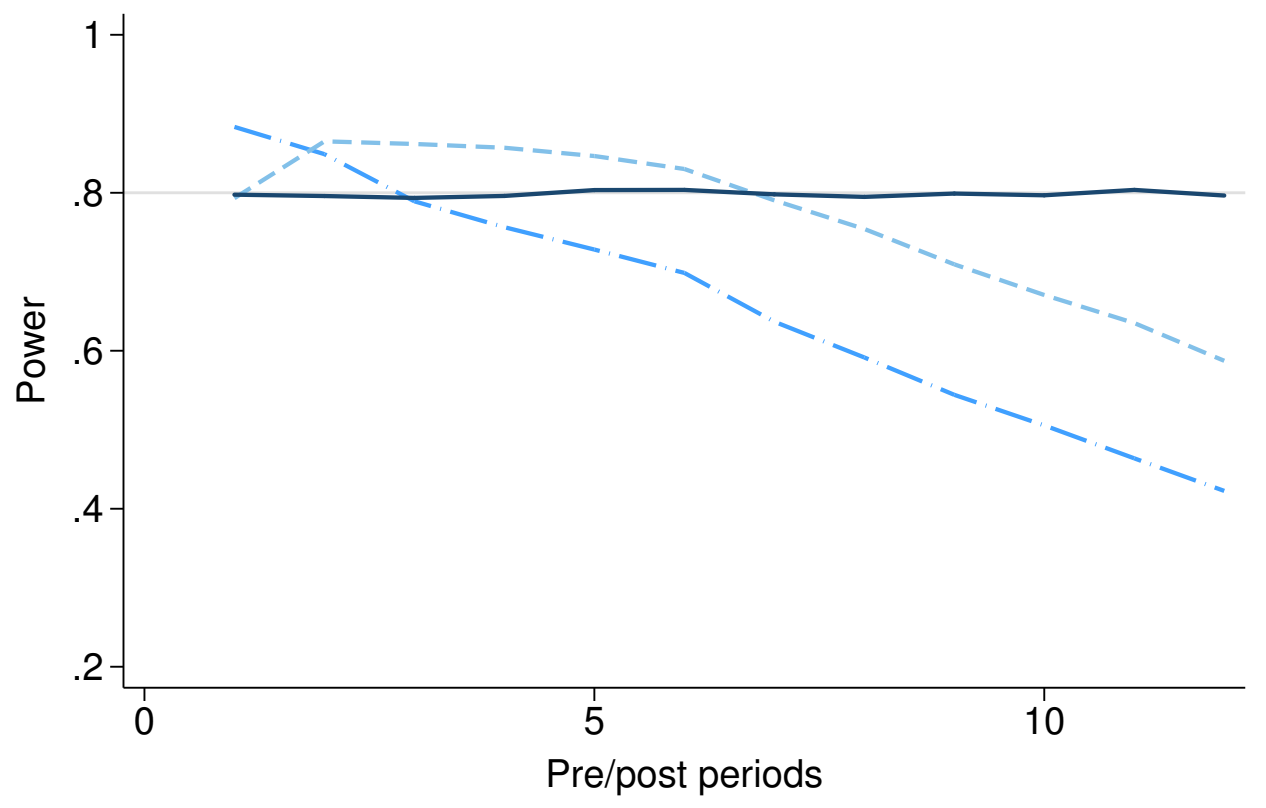

- Frison and Pocock - - AR(1) imposed - Serial-Correlation-Robust

Notes: This figure shows results from Monte Carlo simulations using Bloom et al. (2015) data. Each curve displays the relationship between realized power and the number of pre/post periods used, applying different ex ante assumptions. The long-dashed line uses the Frison and Pocock $(1992)$ formula. The short-dashed line uses the serial-correlation-robust formula, under the assumption that the error structure is $\operatorname{AR}(1)$. We estimate the $\operatorname{AR}(1)$ parameter via Equation (8). The solid line applies the serial-correlation-robust formula, where we non-parametrically generate estimates of $\psi_{\hat{\omega}}^{B}, \psi_{\hat{\omega}}^{A}$, and $\psi_{\hat{\omega}}^{X}$ terms using the Bloom et al. (2015) dataset. All three sets of simulations apply the CRVE ex post, clustering at the individual level. Only the serialcorrelation-robust power calculation formula achieves the desired power of 80 percent, even though the Bloom et al. (2015) data exhibit relatively weak serial correlation.

This confirms our previous finding from simulated data: in a panel data setting with serial correlation, the FP equation does not yield the desired power. By contrast, simulations calibrated

22. We define $\sigma_{\hat{\omega}}^{2}, \psi_{\hat{\omega}}^{B}, \psi_{\hat{\omega}}^{A}$ and $\psi_{\hat{\omega}}^{X}$ to be the estimated analogues of $\sigma_{\omega}^{2}, \psi^{B}, \psi^{A}$, and $\psi^{X}$, where the subscript $\hat{\omega}$ denotes the variance/covariance of residuals rather than errors. Appendix D.1 outlines how to estimate these parameters, and Appendix $\mathrm{B} .2$ provides further details on this simulations. 
with the SCR formula yield 80 percent power, even using real experimental data. These results show that it is possible to design a panel RCT to achieve 80 percent power, so long as the ex ante formula properly accounts for the within-unit correlation structure of the data.

\subsection{Pecan Street data}

Having demonstrated the importance of properly accounting for serial correlation using data from an actual RCT in a developing country setting, we now turn to a much higher-frequency dataset on residential electricity consumption in the United States. Electricity data are particularly wellsuited for studying power calculations for panel RCTs. Hourly electricity data exhibit high withinhousehold autocorrelation, so we should expect to have to adjust our standard errors, and also our power calculations, accordingly. Furthermore, we can aggregate the hourly data by averaging hourly electricity consumption over different time spans, each of which exhibits a distinct correlation structure.

We use publicly available household electricity data from Pecan Street (2016), a research organization based at the University of Texas at Austin, which makes high-resolution energy usage data available to academic researchers. We downloaded a dataset of hourly electricity consumption, measured in kilowatt hours (kWh), from 699 households over 26,888 hours. ${ }^{23}$ We restrict the sample to households that appear in every hour between January 1, 2013 and December 31, 2014 in order to construct a balanced panel, and we drop any household that ever reports zero consumption.24 We are left with a final sample of 97 households over 17,520 hours. We create three additional datasets by collapsing these hourly data into daily, weekly, and monthly averages for each household.

Table 2 presents basic summary statistics, and Figure 4 displays the time series of data for one randomly selected household in our sample at varying levels of aggregation. Unsurprisingly, while mean electricity use is consistent across different collapses of the data, the standard deviation decreases as we move from higher- to lower-frequency datasets.

As observation frequency decreases, the number of observations declines from 1,699,440 total observations over 17,520 periods in the hourly data to 2,328 observations over 24 periods in the monthly dataset. Taken together, this suggests that different frequencies will shed light on the performance of different power calculation approaches, since the hourly data likely has a different correlation structure than the monthly data. Finally, RCTs using energy consumption data are

23. The raw data are available with a login from https://dataport.pecanstreet.org/data/interactive.

24. These observations are likely to be errors. 
becoming increasingly common in economics, making our Pecan Street application relevant to this growing literature.25

Table 2: Summary statistics - Pecan Street

\begin{tabular}{lcccccccc}
\hline \hline DATASET & Mean & Std. Dev. & Min & Max & AR(1) $\hat{\gamma}$ & Households & Periods & Observations \\
\hline Hourly & 1.200 & 1.164 & 0.019 & 13.501 & 0.628 & 97 & 17,520 & $1,699,440$ \\
Daily & 1.200 & 0.789 & 0.082 & 6.013 & 0.651 & 97 & 730 & 70,810 \\
Weekly & 1.198 & 0.739 & 0.105 & 5.175 & 0.713 & 97 & 106 & 10,282 \\
Monthly & 1.197 & 0.712 & 0.169 & 4.296 & 0.654 & 97 & 24 & 2,328 \\
\hline
\end{tabular}

Notes: This table shows summary statistics for electricity consumption in the Pecan Street data. All values are in average $\mathrm{kW}$ of electricity consumed. The raw data are at the hourly level, in $\mathrm{kWh}$. To construct the daily, weekly, and monthly dataset, we average hourly kWh consumption data across the relevant time period. We compute $\hat{\gamma}$ by estimating Equation (8) on residuals from this dataset. In doing so, we cluster standard errors at the household level. All are statistically significant at less than the $1 \%$ level.

We conduct Monte Carlo simulations on all four versions of the Pecan Street data to assess the performance of alternative power calculation assumptions. These follow the exact same procedure as the Bloom et al. (2015) simulations, performed separately for the hourly, daily, weekly, and monthly datasets. We subset consecutive periods to create panels of differing lengths, and we calibrate $\tau^{F P}$ using the FP formula, $\tau^{A R(1)}$ using the SCR formula assuming $\mathrm{AR}(1)$ errors, and $\tau^{S C R}$ using the SCR formula, non-parametrically estimating the $\psi_{\hat{\omega}}$ terms to leverage the full correlation structure of each subsetted dataset ${ }^{26}$ Again, the realized power for each set of simulations is the rejection rate of the null hypothesis, $\tau=0$, across 10,000 iterations.

Figure 5 shows that in all four versions of the Pecan Street data, improperly accounting for serial correlation ex ante yields realized power that sharply deviates from the desired 80 percent. Neither the FP assumption of i.i.d. errors nor an assumed AR(1) structure ex ante manages to account for the serial correlation present in electricity consumption data. As with the Bloom et al. (2015) simulations, we achieve correctly powered experimental designs only by applying the SCR method and accounting for the full covariance structure of the Pecan Street data. This result holds at both high and low temporal frequencies.

25. For example, see Allcott (2011), Jessoe and Rapson (2014), Ito, Ida, and Tanaka (2015), Fowlie, Greenstone, and Wolfram (2015), and Fowlie et al. (2016). There is also a large quasi-experimental literature that uses energy consumption data.

26. See Appendix B.3 for details on the Pecan Street simulations. 
Figure 4: Pecan Street data - Varying levels of aggregation
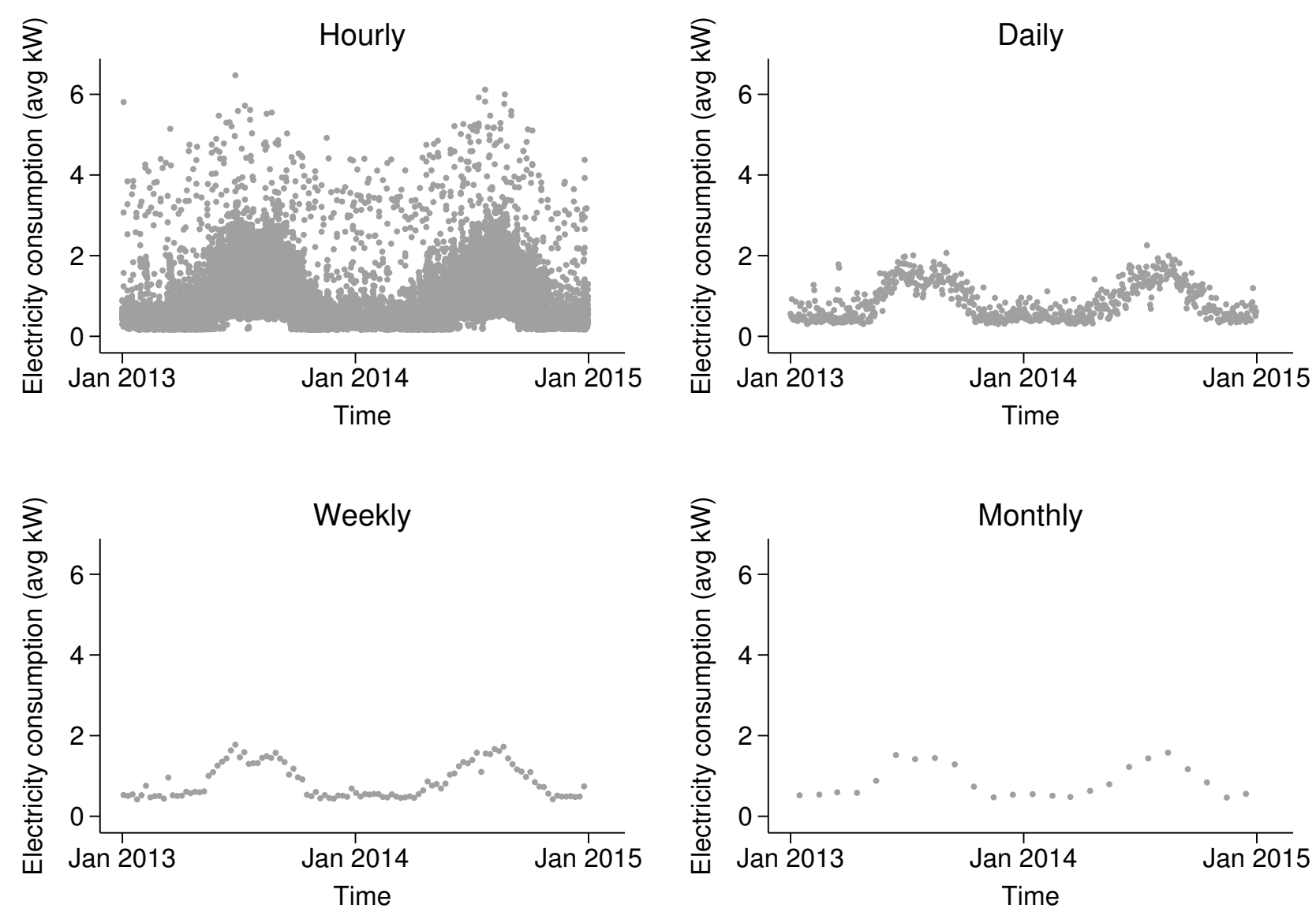

Notes: This figure shows the time series of Pecan Street electricity consumption data for one randomly selected household in our sample. Each panel displays the data at a different level of aggregation. The data are in units of average $\mathrm{kW}$. These data are highly serially correlated: when we estimate an AR(1) model, Equation (8), on residuals from these datasets, we recover the AR(1) parameters of $0.628,0.651,0.713$, and 0.654 for the hourly, daily, weekly, and monthly data, respectively. 
Figure 5: Power simulations for Pecan Street data
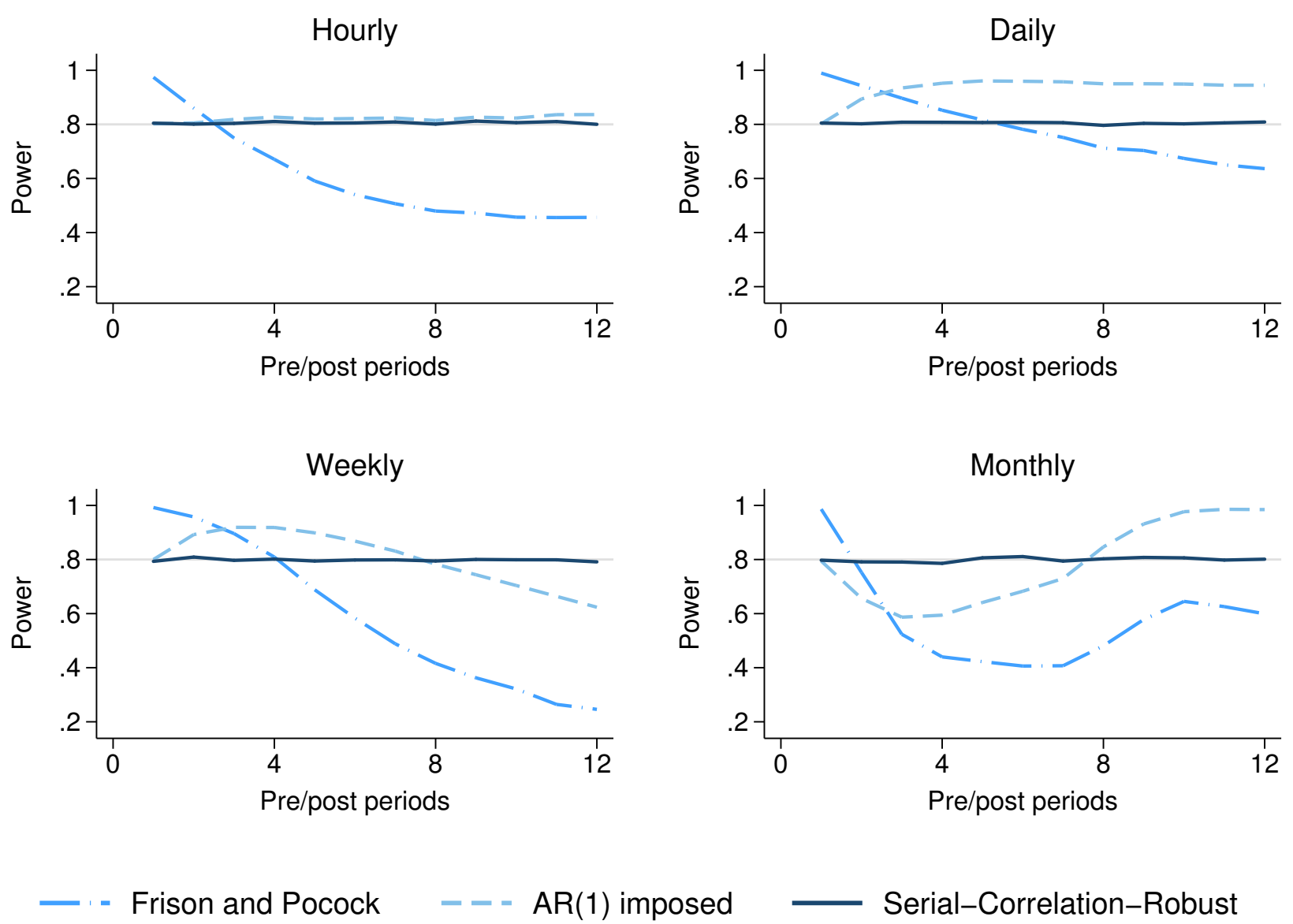

Serial-Correlation-Robust

Notes: This figure shows results from Monte Carlo simulations using the Pecan Street electricity data, collapsed to different levels of aggregation. Each curve displays the relationship between power and the number of pre/post periods used, applying different ex ante assumptions. The long-dashed lines use the Frison and Pocock (1992) formula (Equation (3)). The short-dashed lines use Equation (5) assuming an AR(1) error structure, where we estimate the AR(1) parameters by estimating Equation (8) separately for each dataset. The solid lines apply Equation (5), by estimating separate covariance components of $\psi_{\hat{\omega}}^{B}, \psi_{\hat{\omega}}^{A}$, and $\psi_{\hat{\omega}}^{X}$ terms using residuals from each Pecan Street dataset. All simulations apply the CRVE ex post, clustering at the household level. For each temporal resolution, only the serial-correlation-robust power calculation formula achieves desired power of 80 percent. 


\subsection{Power calculations in real data}

We can use the SCR formula to perform power calculations, which quantify the tradeoff between $M D E$ and $J$ over different panel lengths. In order to operationalize the SCR formula, researchers must assume values for $\sigma_{\omega}^{2}, \psi^{B}, \psi^{A}$, and $\psi^{X}$ that reflect the error structure likely to be present in their (future) experimental datasets. In the best case scenario, researchers will have access to data that are representative of what will be collected in the field, and they can simply calculate these variance and covariance terms from this pre-existing dataset 27 Plugging these estimates into the SCR formula, researchers can evaluate the tradeoffs between different experimental design elements, such as the desired power of the experiment, the number of units to recruit, the number of observations to collect for each unit, and the expected effect size of the intervention.

We perform this procedure on the daily Pecan Street dataset to imitate the design of an experiment that affects household electricity consumption. We do so both under the assumption of uncorrelated errors and also allowing for arbitrary correlations among the error terms of a household using the FP formula and the SCR formula, respectively. For simplicity, we consider only balanced panels of households with the same number of observations before and after the experimental intervention (i.e. $m=r$ ). For each experiment length, we estimate the average $\sigma_{\hat{\omega}}^{2}$ and $\psi_{\hat{\omega}}$ terms from the daily Pecan Street dataset, and we assume constant values for the remaining parameters.28

We plot the results of this exercise in Figure 6. The left panel depicts power calculations using the FP formula, which assumes an i.i.d. error structure. The right panel applies the SCR formula to compute the number of units required for the same set of $M D E \mathrm{~s}$, using our non-parametric estimates of $\psi_{\hat{\omega}}^{B}, \psi_{\hat{\omega}}^{A}$, and $\psi_{\hat{\omega}}^{X}$ to reflect the real error structure of the data. Each curve corresponds to an experiment of a particular length, ranging from $m=r=1$ to $m=r=12$; the curves plot the number of households required to achieve 80 percent power as a function of the $M D E$. For each $M D E$, fewer households are required as the length of the experiment increases. However, the "naive" power calculation always implies a smaller number of households than the SCR power calculation. Hence, if an researcher in this setting applies the CRVE ex post but assumes

27. Appendix D.1 provides details on how to estimate $\sigma_{\hat{\omega}}^{2}, \psi_{\hat{\omega}}^{B}, \psi_{\hat{\omega}}^{A}$, and $\psi_{\hat{\omega}}^{X}$ from pre-existing data, and Appendix E proves that power calculations using estimated parameters recover the same $M D E$ in expectation as those using true parameters. Whether estimating these parameters is plausible will vary across settings. Researchers with implementing partners that have access to large amounts of historical data may use these data to estimate $\sigma_{\hat{\omega}}^{2}$, $\psi_{\hat{\omega}}^{B}$, $\psi_{\hat{\omega}}^{A}$, and $\psi_{\hat{\omega}}^{X}$. On the other hand, this may not be possible for experiments in completely unstudied settings. See Section 6 for more details on how to overcome a lack of pre-experimental data.

28. See Appendix B.4 for details. 
i.i.d. errors ex ante, he will likely include too few households to achieve the desired statistical power.

Figure 6: Analytical power calculations - daily Pecan Street dataset
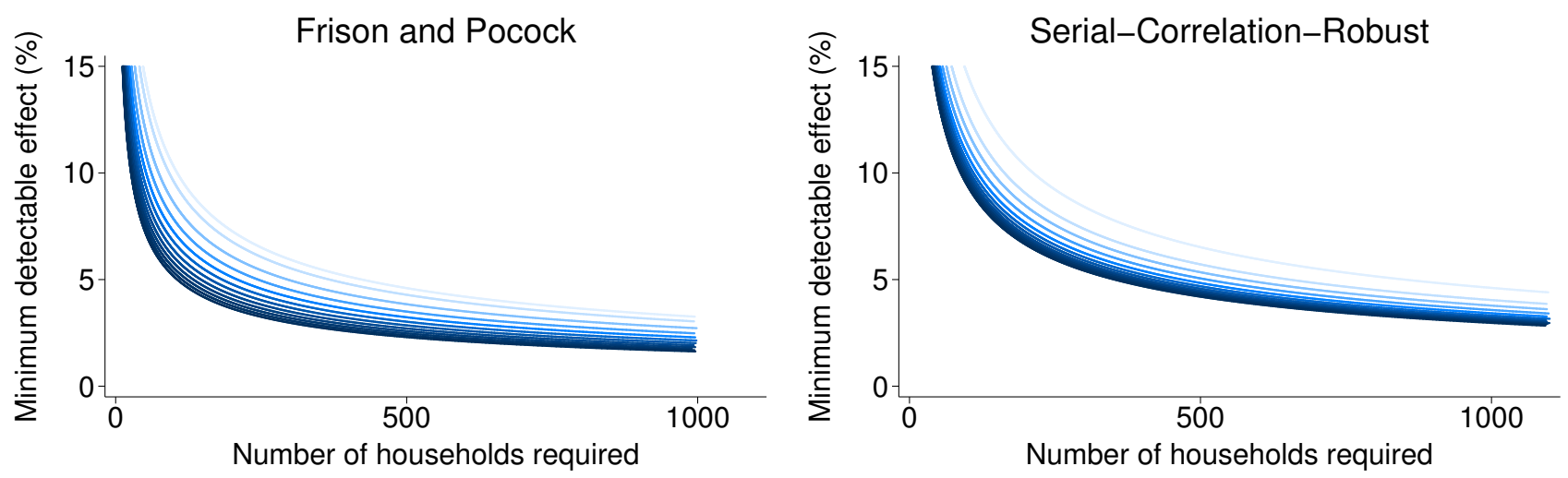

$$
\begin{array}{lllllllllll}
\text { Pre/post periods } & \\
-1-2 & -3 & -4 & -5 & -6 & -7 & -8 & -9 & -10 & -11 & -12
\end{array}
$$

Notes: This figure shows the result of analytic power calculations on Pecan Street electricity data, collapsed to the daily level. Each curve displays the number of units required to detect a given minimum detectable effect with 80 percent power. The individual lines are 80 percent power curves for datasets with varying panel lengths, with the shortest panel (1 pre-period, 1 post-period) in light blue, and the longest panel (12 pre-periods, 12 post-periods) in navy. The left panel shows a power calculation using the Frison and Pocock (1992) formula, which assumes an i.i.d. error structure. The right panel applies the serial-correlation-robust formula, which accounts for the real error structure of the data. Note that, as discussed above, failing to account for the full covariance structure will lead a researcher to dramatically underestimate the sample size required to detect a given effect.

\section{Discussion}

\subsection{Collapsing data}

Above, we demonstrate the importance of considering serial correlation when performing power calculations. Applying the SCR power calculation formula will appropriately account for withinunit correlation, yielding correctly powered experiments. This is analogous to using CRVE standard errors to recover the correct false rejection rate. BDM suggest an alternative way of achieving the correct false rejection rates in the presence of serial correlation: ignore the time-series structure of the data by averaging the pre-treatment and post-treatment data for each unit, then estimate a two-period panel DD regression on this collapsed dataset and apply the OLS variance estimator.

While this approach does recover the desired false rejection rate, simply applying the FP formula to a collapsed dataset will not yield correctly powered experiments. The FP formula for 
collapsed data is:

$$
M D E=\left(t_{1-\kappa}^{J-2}+t_{\alpha / 2}^{J-2}\right) \sqrt{\left(\frac{2}{P(1-P) J}\right) \sigma_{\omega^{C}}^{2}}
$$

where $\sigma_{\omega^{C}}^{2}$ is the variance of $\omega_{i t}^{C}$, the error terms in the collapsed data. In contrast, the SCR formula with data collapsed to one pre- and one post-treatment period is:

$$
M D E=\left(t_{1-\kappa}^{J-2}+t_{\alpha / 2}^{J-2}\right) \sqrt{\left(\frac{2}{P(1-P) J}\right)\left(\sigma_{\omega^{C}}^{2}-\psi^{X}\right)}
$$

where $\psi^{X}$ is the same average covariance term for the uncollapsed data.29 Even when using a collapsed dataset, the FP formula fails to account for the cross-period covariance represented by $\psi^{X}$, and will therefore yield an incorrect $M D E$. While this may seem to contradict the BDM result, the two results are in fact consistent.

Intuitively, to achieve the desired false rejection rate using the OLS variance estimator on collapsed data, it need only be the case that this estimator produce an unbiased estimate of the true variance. Because the collapsed model is a two-period panel, it is isomorphic to a (crosssectional) first-difference model. Since this model has only one observation per unit, it cannot exhibit within-unit correlation over time. This means that the OLS variance estimator is unbiased, and so too is the OLS variance estimator from the equivalent two-period panel model. A researcher aiming to achieve the correct false rejection rate can be agnostic to the actual value of $\operatorname{Var}(\hat{\tau})$; she simply needs to know that the estimator of $\operatorname{Var}(\hat{\tau})$ will be unbiased.

In contrast, for a power calculation, the researcher must know (or have an estimate of) $\operatorname{Var}(\hat{\tau})$. Applying the first-difference model to the collapsed two-period panel, the resulting expression for the variance of the errors of the collapsed-and-first-differenced data is $\sigma_{\Delta \omega^{C}}^{2}=2\left(\sigma_{\omega^{C}}^{2}-\psi^{X}\right)$. That is, we can represent the variance of the collapsed data with a single term, which incorporates both the idiosyncratic variance and serial correlation ${ }^{30}$ To accurately perform power calculations for a

29. The collapsed model begins with Assumptions 6 10, and then averages all $m$ pre-treatment observations and $r$ post-treatment observations for each unit, in order to estimate a two-period pre/post panel with $2 J$ observations. We can express the idiosyncratic error variance of this collapsed model, $\sigma_{\omega^{C}}^{2}$, in terms of uncollapsed parameters, where $\sigma_{\omega C}^{2}=\frac{1}{2}\left[\left(\frac{m+r}{m r}\right) \sigma_{\omega}^{2}+\left(\frac{m-1}{m}\right) \psi^{B}+\left(\frac{r-1}{r}\right) \psi^{A}\right]$. This shows that Equation 11 is identical to the uncollapsed SCR formula in Equation (5). By contrast, Equation (10) differs from the uncollapsed FP formula in Equation (3), because $\sigma_{\omega C}^{2}$ captures a portion of the serial correlation (represented by $\psi^{B}$ and $\psi^{A}$ ). We provide this proof in Appendix A.2.4

30. For example, when compared to data with no serial correlation, a collapsed dataset with positive serial correlation will exhibit smaller first-difference error terms, and hence a smaller error variance. 
collapsed DD model, a researcher may either plug $\sigma_{\Delta \omega^{C}}^{2}$ into the cross-sectional power calculation formula (Equation (2)), or apply the SCR formula which directly incorporates both $\sigma_{\omega C}^{2}$ and $\psi^{X}$. In both cases, generating appropriately powered experiments requires accounting for $\psi^{X}$, and therefore accounting for serial correlation - simply plugging $\sigma_{\omega^{C}}^{2}$ into FP will not yield the desired result.

\section{$5.2 \quad$ ANCOVA}

The vast majority of experiments that have been published in top economics journals, and of ongoing experiments that have been pre-registered at the American Economics Association's RCT registry, estimate treatment effects using analysis of covariance (ANCOVA) methods. These regression specifications include only post-treatment observations, and control for the average pre-treatment level of the outcome variable $\bar{Y}_{i, p r e}=\frac{1}{m} \sum_{t \in m} Y_{i t}$, and post-period fixed effects $\delta_{t}{ }^{31}$

$$
Y_{i t}=\beta+\tau D_{i}+\theta \bar{Y}_{i, p r e}+\delta_{t}+\varpi_{i t}
$$

In a randomized setting, where unit fixed effects are not needed for identification, this method may be preferred to DD because it more efficiently estimates $\hat{\tau}$ (Frison and Pocock (1992)). McKenzie (2012) comments that, with i.i.d. error structures, ANCOVA is always more efficient than the DD model with the same number of periods, but that these gains are eroded as the intracluster correlation coefficient increases.

We examine the relative benefits of ANCOVA in settings with serial correlation, by conducting an analogous simulation exercise to that in Section 3 . As above, we generate data with an $\operatorname{AR}(1)$ parameter governing the error structure. We use the SCR power calculation formula to calibrate $\tau^{M D E}$, such that $\tau^{M D E}$ equals the minimum detectable effect for a panel fixed effects model, and randomly assign treatment at the unit level. Whereas above we estimate $\hat{\tau}$ using a panel DD model, here we estimate $\hat{\tau}$ using Equation (12). We again record whether we reject the null of $\tau=0$, and repeat 10,000 times. We do this twice: once, as above, for varying levels of the $\mathrm{AR}(1)$ parameter $\gamma$;

and also for varying levels of intracluster correlation coefficient $\rho_{v} \cdot{ }^{32}$ Figure 7 presents the results of this exercise, on the same scale as Figure 2 .

31. This model again follows Assumptions 6 10, and then averages all $m$ pre-treatment observations for each unit, in order to estimate a model with $J r$ post-treatment observations only. Note that in most cases, researchers collect one pre-treatment ("baseline") wave of data, and one post-treatment ("follow-up") wave of data. This yields the cross-sectional ANCOVA specification: $Y_{i, p o s t}=\beta+\tau D_{i}+\theta Y_{i, p r e}+\varpi_{i}$.

32. We adjust $\rho_{v}$ by varying $\sigma_{v}^{2}$. See Appendix B.1 for further detail on these ANCOVA simulations. 
Figure 7: The benefits of ANCOVA are limited with serial correlation
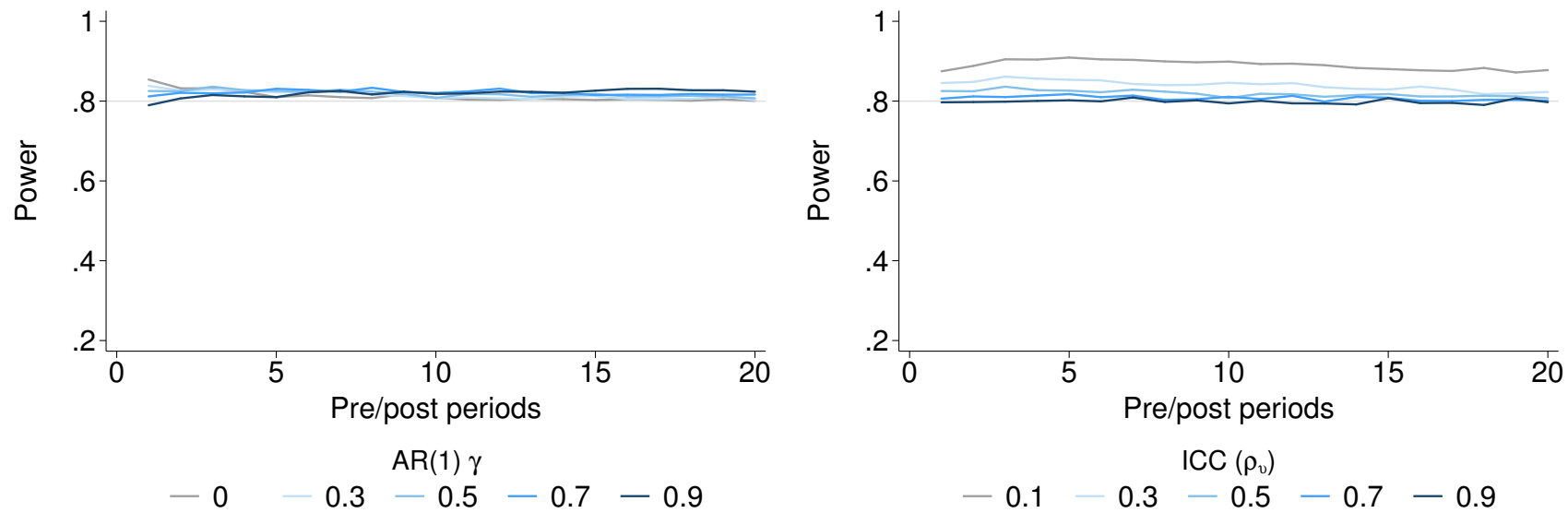

Notes: This figure displays realized power from performing power calculations on data generated with AR(1) processes, where we calibrate the treatment effect size using the serial-correlation-robust power calculation formula from a panel DD model, but estimate the treatment effect using the ANCOVA specification of Equation (12). A realized power of 0.80 indicates that the ANCOVA estimator is exactly as efficient as the DD estimator; power above 0.80 indicates that ANCOVA is more efficient; power below 0.80 indicates that ANCOVA is less efficient. In left panel, we display realized rejections rates for different panel lengths, while varying the level of serial correlation $\gamma$ (setting $m=r$, with $\rho_{v}=0.5$ ). In the right panel, we alternatively vary the intracluster correlation coefficient $\rho_{v}$ for different panel lengths (keeping $m=r$, with $\gamma=0.5$ ). We apply the cluster-robust variance estimator in all cases. While the ANCOVA estimator is generally more efficient than the DD estimator, these gains are relatively small in the presence of serial correlation. As expected, the gains decay as $\rho_{v}$ increases.

Across the range of $\mathrm{AR}(1) \gamma$ values, intracluster correlations, and panel lengths, estimation using ANCOVA provides realized power greater than 80 percent. This means that ANCOVA is indeed more efficient than a panel DD estimator, even in the presence of serial correlation. However, the magnitude of these power gains from ANCOVA is quite modest, especially for moderate levels of $\gamma$ and moderate-to-large $\rho_{v}$. It is intuitive that introducing serial correlation and proper inference will erode the relative efficiency gains of ANCOVA. In a world with i.i.d. errors, much of ANCOVA's efficiency advantage relative to DD results from its use of just one degree of freedom in predicting the post-treatment outcome with pre-treatment data (i.e. in estimating $\theta$ ), as opposed to $J$ degrees of freedom for $J$ unit fixed effects in a DD model. In settings with serial correlation, however, both ANCOVA and DD require the CRVE with $J$ degrees of freedom, thereby eroding the degrees-offreedom advantage of ANCOVA. Our results suggest that ANCOVA only marginally improves the efficiency of DD in the presence of serial correlation. 


\subsection{Trading off $J$ for $m$ and $r$}

Recruiting participants, administering treatment, and collecting data are all costly. Implementation costs are often the limiting factor in study size. Power calculations enable researchers to calibrate experimental design parameters to a particular $M D E$. We can use the power calculation framework to conceptualize the optimal design of a panel RCT given a budget, by couching it in a simple constrained optimization problem of the following form:

$$
\min _{P, J, m, r} M D E(P, J, m, r) \quad \text { s.t. } \quad p_{T} P J+p_{C}(1-P) J+p_{m} J m+p_{r} J r \leq B
$$

Here, $p_{T}$ and $p_{C}$ are the costs of including each treated and control unit in the experiment, respec-

tively; $p_{m}$ and $p_{r}$ are the costs of collecting one pre- and post-treatment observation, respectively; and $B$ is the experiment's budget.

The budget constraint creates a fundamental tradeoff between including additional units and including additional time periods in the experiment, since each comes at a cost. This tradeoff arises not only from differences in these costs, but also from differences in the marginal effects of additional units and time periods on the $M D E$. These marginal effects can be calculated by taking the partial derivative of the $M D E$ formula with respect to each of these design parameters. Using the SCR power calculation formula, the "elasticities" of the $M D E$ with respect to number of units and number of time periods are:

$$
\begin{aligned}
& \frac{\partial M D E / M D E}{\partial J / J}=-\frac{1}{2} \\
& \frac{\partial M D E / M D E}{\partial m / m}=-\frac{1}{2}\left[\frac{\frac{\sigma_{\omega}^{2}}{m}-\frac{\psi^{B}}{m}-(m-1) \frac{\partial \psi^{B}}{\partial m}+2 m \frac{\partial \psi^{X}}{\partial m}}{\left(\frac{m+r}{m r}\right) \sigma_{\omega}^{2}+\left(\frac{m-1}{m}\right) \psi^{B}+\left(\frac{r-1}{r}\right) \psi^{A}-2 \psi^{X}}\right] \\
& \frac{\partial M D E / M D E}{\partial r / r}=-\frac{1}{2}\left[\frac{\frac{\sigma_{\omega}^{2}}{r}-\frac{\psi^{A}}{r}-(r-1) \frac{\partial \psi^{A}}{\partial r}+2 r \frac{\partial \psi^{X}}{\partial r}}{\left(\frac{m+r}{m r}\right) \sigma_{\omega}^{2}+\left(\frac{m-1}{m}\right) \psi^{B}+\left(\frac{r-1}{r}\right) \psi^{A}-2 \psi^{X}}\right]
\end{aligned}
$$

There is a constant elasticity of $M D E$ with respect to $J$ of -0.5 , meaning that a 1 percent increase in the number of units always yields a 0.5 percent reduction in the MDE. However, the elasticity of $M D E$ with respect to $m$ and $r$ varies as a function of the error structure and the number of time periods. ${ }^{33}$ For some parameter values, this elasticity can be positive, such that increasing

33. Note that $J, m$, and $r$ must all be integer-valued, hence these derivatives serve as continuous approximations of discrete changes in these parameters. Likewise, the partial derivatives of $\psi^{B}, \psi^{A}$, and $\psi^{X}$ with respect to $m$ and 
the length of the experiment would actually increase the $M D E$. This may seem counter-intuitive, but adding time periods can reduce the average covariance between pre- and post-treatment observations, $\psi^{X}$, which introduces more noise in the estimation of pre- vs. post-treatment difference. For relatively short panels with errors that exhibit strong serial correlation, this effect can dominate the benefits of collecting more time periods ${ }^{34}$

Figure 8: Analytical power calculations with increasing panel length
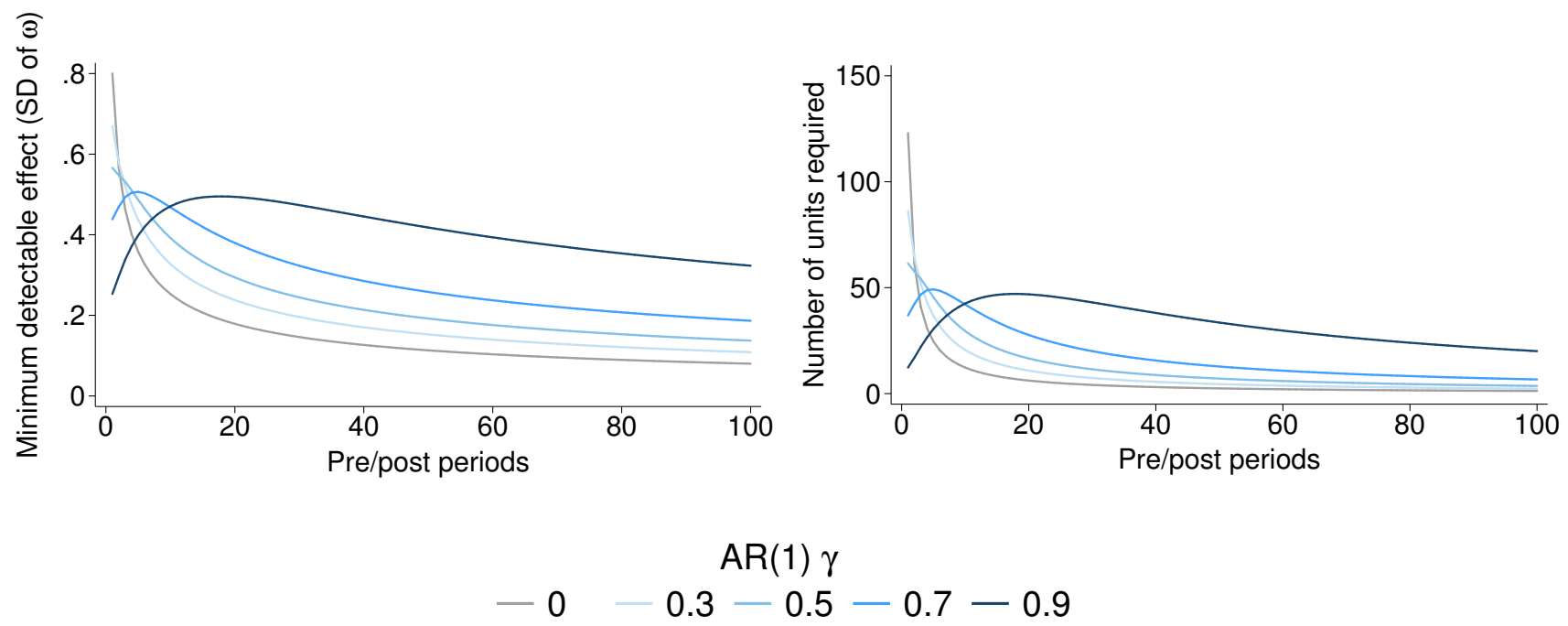

Notes: This figure displays the results of analytical power calculations using the serial-correlation-robust formula for varying $\mathrm{AR}(1)$ parameters. The left panel shows the tradeoff between the minimum detectable effect $(M D E)$ and the number of time periods $(m=r)$ for varying levels of serial correlation, holding the number of units fixed at $J=100$ and normalizing $M D E$ by the standard deviation of $\omega_{i t}$. At low levels of $\gamma$, the $M D E$ declines monotonically in $m$ and $r$. However, for higher $\gamma$, increasing $m$ and $r$ actually increases the $M D E$ when $m=r$ is relatively small and decreases the $M D E$ when $m=r$ is relatively large. The right panel shows the relationship between the number of units $(J)$ and number of pre/post periods $(m=r)$ required to detect an $M D E$ equal to one standard deviation of $\omega_{i t}$. Similarly, for low levels of serial correlation, the trade-off between $J$ and $m=r$ is monotonic. However, as $\gamma$ increases, adding periods in short panels necessitates a greater number of units to achieve the same $M D E$, while adding periods in longer panels means that fewer units are required to achieve the same $M D E$.

Figure 8 illustrates the fact that additional time periods may either increase or decrease statistical power. The left panel plots the $M D E$ of an experiment as a function of the number of pre- and post-treatment waves, holding the number of units $J$ constant. We normalize the $M D E$

$r$ are not technically defined, as these covariance terms are averaged over discrete numbers of periods (as shown in Equations A33-A35 of Appendix A.1.1.

34. Researchers may also adjust $P$ to make an experimental design more cost effective. An RCT will have the lowest $M D E$ at $P=0.5$, but if control units are cheap compared to treatment units, the same power may be achieved at lower cost by decreasing $P$ and increasing $J$. See Duflo, Glennerster, and Kremer (2007) for more details. We also typically consider $\alpha$ and $\kappa$ to be fixed "by convention." While $\alpha$ is the product of research norms, and therefore relatively inflexible, researchers may want to adjust $\kappa .1-\kappa$ is the probability of being unable to distinguish a true effect from 0. In lab experiments which are cheaply replicated, researchers may accept $\kappa<0.80$, whereas in large, expensive field experiments that can only be conducted once, researchers may instead wish to set $\kappa>0.80$. Researchers may also choose to size their experiments such that they achieve a power of 80 percent for the smallest economically meaningful effect, even if they expect the true MDE to be larger. 
to be in units of $\sigma_{\omega}$. The right panel depicts the tradeoff between additional units and additional time periods, by plotting the combinations of $J$ and $m=r$ that yield an $M D E$ equal to $\sigma_{\omega}$. To analytically construct these curves, we use the SCR formula and assume that the error structure is $\operatorname{AR}(1)$ with varying $\gamma$ values.

At low to moderate levels of serial correlation, increasing the panel length always reduces the $M D E$ for a given $J$, and likewise reduces the $J$ required to achieve a given $M D E$. However, at higher levels of serial correlation, this relationship is no longer monotonic. In fact, for $\gamma \geq 0.6$, marginally increasing $m$ or $r$ in a relatively short panel actually increases the $M D E$ for a given $J$, and likewise increases the $J$ required to achieve a given $M D E$. While such non-monotonicities only occur in settings with a high degree of serial correlation, we recover $\hat{\gamma}>0.6$ for all four versions of the Pecan Street dataset. This suggests that for experiments using highly correlated data, additional periods of data might decrease statistical power if the panel is not sufficiently long.

\section{Power calculations in practice}

\subsection{A simulation-based approach}

In this paper, we develop an analytical framework for performing power calculations in a DD model with panel data that have a non-i.i.d. error structure. Shifting from the i.i.d. model to a non-i.i.d. model increases the number of parameters required to calibrate a DD power calculation formula. This reveals a fundamental challenge of analytical power calculations: more complex experimental designs and DGPs require more complex treatment effect estimators, which in turn have analytic variance expressions that are increasingly difficult to derive and parameterize. For example, if we relax the assumption of randomization and instead consider a quasi-experimental DD design, where cross-sectional correlations remain important, the expression for $\operatorname{Var}(\hat{\tau})$ includes 13 separate $\psi$ parameters - each of which would need to be non-parametrically estimated to fully characterize the error structure of the data and conduct an analytical power calculation 35

In light of these challenges, we recommend performing power calculations via simulation rather than by using analytic formulas in cases where researchers have access to a pre-existing dataset that is representative of their future experimental data. Simulation-based power calculations follow a straightforward Monte Carlo process, where each simulation implements the proposed estimating

35. See Appendix A.2.3 for the full derivation and resulting power calculation equation. 
equation over a range of assumed treatment effect sizes $(\tau)$, numbers of units $(J)$, proportion of units treated $(P)$, and panel lengths $(m$ and $r){ }^{36}$ Calculating the average rejection rates of the null hypothesis $\tau=0$ over all simulations will reveal the statistical power of each parameterization, and researchers can compare power across parameterizations to find their preferred values of $P, J, m$, and $r$.

Importantly, simulation-based power calculations do not require researchers to estimate $\operatorname{Var}(\hat{\tau})$ as a function of the underlying error structure in the data. This allows for greater flexibility in selecting research designs, and easily facilitates comparisons across alternative estimating equations. For example, a simulation-based power calculation for a proposed experiment using hourly electricity consumption data could compare the standard DD specification with individual and time fixed effects to an alternative specification that also includes group-specific time trends, without needing to formally derive an expression for $\operatorname{Var}(\hat{\tau})$ under this alternative model.

Simulation-based power calculations allow researchers to leverage any representative pre-existing data that may exist, and our analytical results provide key intuition for interpreting this simulation output. Given that power calculations via simulation are computationally intensive and necessitate a grid search over the full space of candidate design parameters, researchers may begin by using analytical power calculation formulas to narrow the range of plausible parameter values. In the absence of representative data ex ante, researchers may apply analytical techniques (with appropriate sensitivity analyses) to inform experimental design. It may still be possible to calibrate the variance-covariance parameters in the serial-correlation-robust power calculation formula, even if the ideal pre-existing dataset is not available.

\subsection{Practical challenges}

All power calculations are a combination of art and science (Duflo, Glennerster, and Kremer (2007)). Even in cases where researchers are able to simulate power calculations using a large pre-existing dataset, they must assume that these data are representative of the underlying DGP governing their future experiment ${ }^{37}$ Imperfect pre-existing datasets can be used to inform ex ante experimental

36. For each simulation, the researcher re-randomizes $P J$ units into treatment, adds $\tau$ to treated units' outcomes for all post-treatment periods, and estimates $\hat{\tau}$ using her preferred variance estimator. We provide further guidance on simulation-based power calculations in Appendix D.2.

37. It is always possible that experimental data will differ systematically from a seemingly representative preexisting dataset, due to either cross-sectional sampling differences or exogenous time trends affecting the outcome of interest. 
design, even if they are less representative than desired, and especially if they are smaller than the expected size of the experiment. For example, if a pre-existing dataset has too few crosssectional units, researchers can bootstrap these units to construct a larger dataset for the purposes of simulating power calculations. Likewise, if a pre-existing dataset has too few time periods, it may be possible to estimate the error structure in this short panel in order to bound estimates of the $\psi^{B}, \psi^{A}$, and $\psi^{X}$ covariance parameters and apply the analytical formula 38

In some settings, researchers may have no pre-existing data whatsoever, meaning that power calculations will require substantial guesswork. In this scenario, we recommend that researchers apply our SCR formula analytically, assuming a range of reasonable parameters. It may be possible to benchmark these "reasonable" parameter values to previous research, coupled with appropriate sensitivity analyses.

While accounting for non-i.i.d. errors increases the number of parameters needed to perform power calculations, ignoring the potential for serially correlated errors can significantly undermine an experiment's chances of success. Hence, it is important to consider the error structure ex ante, even in the absence of pre-existing data. In any case, we recommend that researchers perform power calculations under a range of assumed parameter values, in order to test the sensitivity of their experiment's $M D E$ to different assumptions about the data generating process and to experimental design parameters.

\section{Conclusion}

Randomized experiments are costly, and it is important that researchers avoid underpowered experiments that are not informative, as well as dramatically overpowered experiments that waste resources. Statistical power calculations help researchers to calibrate the sample size of experiments ex ante, such that they are likely to collect enough data to detect treatment effects of a meaningful size, while also unlikely to collect excessive amounts of costly data. As data collection becomes easier and cheaper, panel data are becoming increasingly common in randomized experiments. Temporally disaggregated data allow researchers to ask new questions, and to apply a wider range of empirical methods to answer these questions (McKenzie (2012)).

In this paper, we identify a fundamental mismatch between existing ex ante power calculation techniques and ex post inference in panel data settings. We develop new tools to incorporate serial

38. See Appendix D.3 for further detail on strategies to overcome shortcomings in pre-existing data. 
correlation into the design of panel RCTs. We derive the variance of a panel difference-in-differences estimator allowing for arbitrary within-unit correlation, which we use to update the conventional differences-in-differences power calculation formula derived by Frison and Pocock (1992). This new "serial-correlation-robust" formula is consistent with the CRVE variance estimator, which has become standard practice in ex post analysis of panel RCTs. We use Monte Carlo analyses to demonstrate that our updated power calculation formula achieves the desired statistical power, whereas the conventional formula is likely to produce either dramatically underpowered or overpowered experiments in the presence of serially correlated errors. These results hold in real data from a panel RCT in China, and for household electricity consumption data similar to that used in panel RCTs in the energy economics literature.

Our work highlights the need to carefully consider the assumptions that will enter ex post analysis when calibrating the design of experiments ex ante. The serial-correlation-robust power calculation framework allows researchers to conduct power calculations that correctly account for within-unit correlation, and provides intuition about these calculations under non-i.i.d. errors. We extend the main results by demonstrating that serial correlation matters, even when collapsing data or implementing ANCOVA methods; providing researchers with a framework for trading off units for effect sizes that takes cost into account; and discussing implementation of power calculations in practice ${ }^{39}$ Ultimately, we provide researchers with new tools for experimental design that properly account for serial correlation in panel data settings, which enable the development of well-powered experiments with multiple waves of data.

39. We have an accompanying software package, pcpanel, which makes our power calculation method easily accessible and user-friendly. The STATA version is currently is available from ssc, with an $\mathrm{R}$ version, which will be available from CRAN, to follow shortly. 


\section{References}

Allcott, Hunt. 2011. "Social Norms and Energy Conservation." Journal of Public Economics 95 (9): 1082-1095.

Angrist, Joshua D., and Jorn-Steffen Pischke. 2010. "The Credibility Revolution in Empirical Economics: How Better Research Design is Taking the Con out of Econometrics." Journal of Economic Perspectives 24 (2): 3-30.

Arellano, Manuel. 1987. "Computing Robust Standard Errors for Within-Group Estimators." Oxford Bulletin of Economics and Statistics 49 (4): 431-34.

Athey, Susan, and Guido W. Imbens. 2016a. "The Econometrics of Randomized Experiments." Working Paper.

_. 2016b. "The State of Applied Econometrics - Causality and Policy Evaluation." Working Paper.

Baird, Sarah, J. Aislinn Bohren, Craig McIntosh, and Berk Ozler. 2014. "Designing Experiments to Measure Spillover Effects." World Bank Policy Research Working Paper WPS 6824.

Bertrand, Marianne, Esther Duflo, and Sendhil Mullainathan. 2004. "How Much Should We Trust Differences-In-Differences Estimates?" The Quarterly Journal of Economics 119 (1): 249-275.

Blattman, Christopher, Nathan Fiala, and Sebastian Martinez. 2014. "Generating Skilled SelfEmployment in Developing Countries: Experimental Evidence from Uganda." The Quarterly Journal of Economics 129 (2): 697-752.

Bloom, Howard S. 1995. "Minimum Detectable Effects: A Simple Way to Report the Statistical Power of Experimental Designs." Evaluation Review 19 (5): 547-556.

Bloom, Nicholas, Benn Eifert, Aprajit Mahajan, David McKenzie, and John Roberts. 2013. "Does Management Matter? Evidence from India." The Quarterly Journal of Economics 128 (1): 151.

Bloom, Nicholas, James Liang, John Roberts, and Zhichun Jenny Ying. 2015. "Does Working from Home Work? Evidence from a Chinese Experiment." The Quarterly Journal of Economics 130 (1): 165-218.

Cameron, A. Colin, and Douglas L. Miller. 2015. "A Practitioner's Guide to Cluster-Robust Inference." Journal of Human Resources 50 (2): 317-372.

Campbell, Cathy. 1977. "Properties of Ordinary and Weighted Least Square Estimators of Regression Coefficients for Two-Stage Samples." Proceedings of the Social Statistics Section, American Statistical Association: 800-805.

Card, David, Stefano DellaVigna, and Ulrike Malmendier. 2011. "The Role of Theory in Field Experiments." Journal of Economic Perspectives 25 (3): 39-62.

Cohen, Jacob. 1977. Statistical Power Analysis for the Behavioral Sciences. New York, NY: Academic Press.

Duflo, Esther, Rachel Glennerster, and Michael Kremer. 2007. "Using Randomization in Development Economics Research: A Toolkit." Chap. 61 in Handbook of Development Economics, edited by Paul T. Schultz and John A. Strauss, 3895-3962. Volume 4. Oxford, UK: Elsevier. 
Fowlie, Meredith, Michael Greenstone, and Catherine Wolfram. 2015. "Do Energy Efficiency Investments Deliver? Evidence from the Weatherization Assistance Program." NBER Working Paper 21331.

Fowlie, Meredith, Catherine Wolfram, C. Anna Spurlock, Annika Todd, Patrick Baylis, and Peter Cappers. 2016. "Default Effects, Follow-on Behavior and Welfare in Residential Electricity Pricing Programs." Working Paper.

Frison, L., and S. J. Pocock. 1992. "Repeated Measures in Clinical Trials: Analysis Using Mean Summary Statistics and its Implications for Design." Statistics in Medicine 11 (13): 16851704.

Glennerster, Rachel, and Kudzai Takavarashi. 2013. Running Randomized Evaluations: A Practical Guide. Princeton, NJ: Princeton University Press.

Ito, Koichiro, Takanori Ida, and Makoto Tanaka. 2015. "The Persistence of Moral Suasion and Economic Incentives: Field Experimental Evidence from Energy Demand." NBER Working Paper 20910.

Jessoe, Katrina, and David Rapson. 2014. "Knowledge Is (Less) Power: Experimental Evidence from Residential Energy Use." American Economic Review 104 (4): 1417-1438.

McKenzie, David. 2012. "Beyond Baseline and Follow-up: The Case for More T in Experiments." Journal of Development Economics 99 (2): 210-221.

Moulton, Brent. 1986. "Random group effects and the precision of regression estimates." Journal of Econometrics 32 (3): 385-397.

Murphy, Kevin, Brett Myors, and Allen Wolach. 2014. Statistical Power Analysis: A Simple and General Model for Traditional and Modern Hypothesis Tests. 4th ed. New York, NY: Routledge.

Pecan Street. 2016. "Dataport." https://dataport.pecanstreet.org/.

Rubin, Donald B. 1974. "Estimating Causal Effects of Treatments in Randomized and Nonrandomized Studies." Journal of Educational Psychology 66 (5): 688-701.

White, Halbert. 1984. Asymptotic Theory for Econometricians. 1st ed. San Diego, CA: Academic Press. 